\title{
Improving Postural Stability among Amputees by Tactile Sensory Substitution
}

\section{Lijun Chen}

Peking University

\section{Yanggang Feng}

Peking University

\section{Baojun Chen}

Peking University

\section{Qining Wang}

Peking University

Kunlin Wei ( $\square$ wei.kunlin@pku.edu.cn )

Peking University https://orcid.org/0000-0001-5098-3808

\section{Research}

Keywords: sensory substitution, amputees, postural control, intelligent prosthesis

Posted Date: April 6th, 2021

DOl: https://doi.org/10.21203/rs.3.rs-364267/v1

License: (c) (1) This work is licensed under a Creative Commons Attribution 4.0 International License. Read Full License 
$1 \quad$ Improving Postural Stability among Amputees by Tactile Sensory Substitution

$6 \quad{ }^{1}$ School of Psychological and Cognitive Sciences, Peking University, Beijing, China;

$7 \quad{ }^{2}$ Beijing Key Laboratory of Behavior and Mental Health, Beijing, China;

$8 \quad{ }^{3}$ Department of Advanced Manufacturing and Robotics, College of Engineering, Peking

9 University, Beijing, China;

$10{ }^{4}$ Key Laboratory of Machine Perception, Ministry of Education, Beijing, China;

$11{ }^{5}$ Peking-Tsinghua Center for Life Sciences, Beijing, China.

12

13 Corresponding author $(*)$ :

14 Kunlin Wei (wei.kunlin@pku.edu.cn)

15 School of Psychological and Cognitive Sciences, Peking University, 5 Yiheyuan Road, 


\section{ABSTRACT}

24

\section{Background}

For lower-limb amputees, wearing a prosthetic limb helps restore their motor abilities for daily activities. However, the prosthesis's potential benefits are hindered by limited somatosensory feedback from the affected limb and its prosthesis. Previous studies have examined various sensory substitution systems to alleviate this problem; the prominent approach is to convert foot-ground interaction to tactile stimulations. However, positive outcomes for improving amputees' postural stability are still rare. We hypothesize that the intuitive design of tactile signals based on psychophysics shall enhance the feasibility and utility of real-time sensory substitution for lower-limb amputees.

\section{Methods}

We designed a wearable device consisting of four pressure sensors and two vibrators and tested it among the unilateral transtibial amputees $(n=7)$ and the able-bodied $(n=8)$. The realtime measurements of foot pressure were fused into a single representation of foot-ground interaction force, which was encoded by varying vibration intensity of the two vibrators attached to the participants' forearm. The layout of vibrators was spatially congruent with the foot force sensors' placement; the vibration intensity followed a logarithmic function of the force representation, in keeping with principles of tactile psychophysics. The participants 
41 were tested with a classical postural stability task in which visual disturbances perturbed their

42 quiet standing.

\section{Results}

44 With a brief familiarization of the system, the participants exhibited better posture

45 stability against visual disturbances when switching on sensory substitution than without. The

46 body sway was substantially reduced, as shown in head movements and excursions of the center of pressure. The improvement was present for both amputees and able-bodied controls and was particularly pronounced in more challenging conditions with larger visual disturbances.

\section{Conclusions}

52 improve postural stability for lower-limb amputees. The intuitive design of the mapping

53 between the foot-ground interaction force and the tactile signals is essential for the user to

54 utilize the surrogated tactile signals for postural control, especially for situations that their

55 postural control is challenged.

57 KEYWORDS: sensory substitution, amputees, postural control, intelligent prosthesis 


\section{Introduction}

62 For amputees, wearing a prosthetic limb can help restore their motor functions and improve

63 life quality. For fluent and adaptive motor performance, the nervous system employs a close

64 sensorimotor loop where efferent motor outputs are continuously coupled with afferent

65 sensory feedback (1). The development of typical lower-limb prosthetics, even those robotic

66 prosthetics with actuation, focuses on the efferent control, i.e., controllability and usability of

67 the prosthetic limb without providing the missing sensory feedback caused by amputation (2).

68 Studies on intelligent lower-limb prosthesis have made impressive progress in adaptive

69 control of the knee and ankle joints for walking (3-7) and even used electromyography of

70 residual limb muscles to adjust the force or torque of prosthetic joints (8-10). Essentially,

71 these studies aimed to realize fluent control of the robotic prosthetics with efficiency and

72 precision. However, supplying suitable afferent feedback for lower-limb prosthesis users is

73 still understudied.

74 The lower-limb amputee lacks direct foot contact with the ground and the feedback from foot

75 mechanoreceptors, critical for balance control (11). With a broken sensorimotor loop,

76 amputees often show poor balance and gait function with fear of falling and a high prevalence

77 of falls $(12,13)$. When an amputee wears a prosthesis, the residue limb of the amputee

78 physically interacts with the prosthetic sockets and provides limited haptic feedback that

79 indirectly reflects foot-ground interaction. Augmenting this essential feedback for prosthesis

80 wearers has the potential to close the sensorimotor control loop and subsequently improve

81 their gait control and postural stability $(14,15)$. 
83 system via an alternative, intact sensory channels. For example, auditory and haptic feedback

84 has been used to surrogate visual feedback for the blinded to explore the surroundings (16).

85 For upper-limb amputees, sensory substitution has been shown to provide effective sensory

86 feedback for controlling robotic arms (17). Previous researchers have also explored the

87 coding of movement-related information via visual, auditory, or tactile channels for lower-

88 limb amputees. For example, Zambarbieri, Schmid (18) used a pressure-sensing insole to

89 estimate the center of pressure $(\mathrm{CoP})$ underneath the foot and visually present the estimate to

90 the participant. This method is apparently impractical since the processing of the surrogated

91 visual information is cognitively demanding and thus limits the benefit of sensory substitution

92 for gait and postural control, which are typically controlled with minimal cognitive load.

93 Other researchers have also used auditory feedback to deliver gait balance information and

94 demonstrated a positive effect on gait asymmetry $(19,20)$. However, the auditory solutions

95 are also impractical given their high demands on cognitive resources and their surroundings'

96 quietness. Thus, it is understandable that most researchers have turned to tactile sensory

97 substitution for prosthetic control. The tactile feedback is typically delivered by electrotactile

98 stimulation $(21,22)$ or vibrotactile stimulation $(23-27)$, the latter being the more favorable

99 one for amputees since it is more comfortable to wear (28).

100 However, the potential benefits of tactile sensory substitution for lower-limb amputees have

101 not been firmly established. Fan, Culjat (24) developed a tactile device consisting of four

102 pneumatically controlled balloon actuators that pressed against the residue thigh of the 
103 amputated leg with a force magnitude linearly scaled by the pressure measurements from the

104 insole of the prosthesis. They found that, based on the data from a single transtibial amputee,

105 the intensity and the order of pressing forces applied by the balloon actuators could be

106 estimated with decent accuracy $(24,25)$. However, they did not assess the efficacy of the

107 system in any motor task with prosthesis use. Furthermore, the large size of the balloon

108 actuators might prevent its wide use in the amputee population. Plauché, Villarreal (29) and

109 Crea, Cipriani (23) used similar instrumented insoles but applied electrotactile vibrations on

110 the thigh to inform the amputee about the phase transitions of gait. However, these studies

111 only tested the device on non-disabled participants to show its feasibility and efficacy. The

112 only study that actually examined the postural balance in amputees with tactile sensory

113 substitution returned mixed or little beneficial results (26). This study again placed four

114 vibrators on the thigh to applied tactile stimuli contingent on the measurement of four plantar

115 pressure sensors placed in the insole. The vibration intensity changed in proportion to the

116 amount of plantar pressure. Three separate tasks were used to assess its effect on postural

117 balance, including quiet standing, reaching to a visual target with a cursor representation of

$118 \mathrm{CoP}$, and continuously tracking an oscillatory target with the CoP cursor. Among dozens of

119 performance variables, only the reaction time of the CoP reaching task showed improvement

120 with sensory substitution among amputees. In fact, the mediolateral range of CoP movements,

121 negatively correlated with postural stability during quiet standing, increased with sensory

122 substitution. In sum, previous researches on lower-limb amputees either did not examine the 
123 effect of tactile sensory substitution on balance performance or failed to provide a convincing

124 beneficial effect.

125 These findings appear discouraging for the application of tactile sensory substitution in lower-

126 limb amputees. However, recent studies have shown that foot-ground contact feedback

127 delivered by directly stimulating the afferent nerves in the residuum of transtibial amputees

128 can improve their postural stability and gait $(30,31)$. Furthermore, extra tactile feedback also

129 has been shown to improve postural control among vestibular patients (32-34) and patients

130 with Parkinson's disease (34). We thus hypothesize that proper design of the vibrotactile

131 system can enhance standing balance among lower-limb amputees. Previous approaches can

132 be improved in at least two technical aspects. First, the spatial correspondence between the

133 foot's missing sensation and the surrogate tactile signal shall be intuitive to the prosthesis

134 user. For instance, most studies measured plantar pressure at four locations underneath the

135 foot and mapped it onto vibrotactile stimulations applied at four locations on the thigh (23,

$13626,29)$. However, the spatial layout of the vibrators was around the thigh. It is conceivable

137 that the motor system needs considerable training before incorporating the spatially-

138 incongruent tactile information into the sensorimotor control loop. However, none of those

139 above studies provided any dedicated training session. The solution is either giving

140 participants extensive training with the device, or making the vibrotactile stimulus more

141 intuitive to learn, or both. Second, previous studies typically encoded tactile stimulation as a

142 linear function of the magnitude of plantar pressure. However, human tactile perception is a

143 nonlinear function of stimulus amplitude, i.e., perceptual discrimination of changes 
144 deteriorates with stimulus intensity $(35,36)$. Thus, a high-intensity tactile stimulus is less

145 informative. Currently, this nonlinearity in tactile perception has not been taken into

146 consideration to enhance the efficacy of sensory substitution. One of our previous studies also

147 confirmed that amputees have more difficulty distinguishing the intensities of tactile stimuli

148 than locating them on the skin (37).

149 In the present study, we designed an intuitive tactile stimulation system to provide real-time

150 feedback on plantar pressure. We tested its efficacy in improving postural stability among

151 amputees and the non-disabled. We measured plantar pressure at four insole locations and

152 mapped it nonlinearly to tactile intensity. Critically, to make the learning of sensory

153 substitution easy and intuitive, our system only encodes CoP excursions in the anteroposterior

154 direction, a more critical direction of instability among amputees than other directions (38).

155 Thus, we only needed to use two vibrators and aligned them on the forearm's longitudinal

156 axis, which corresponds to the anteroposterior body sway. Previous studies usually used quiet

157 standing or treadmill walking when comparing postural control ability between lower-limb

158 amputees and non-disabled participants (38-41). We similarly used quiet standing but

159 examined its stability under visual disturbance with the classical moving-room paradigm (42).

160 We found that after minimal training with the system, both amputees and the non-disabled

161 improved their postural stability under visual disturbances. The improvement was particularly

162 large when the visual disturbances are more challenging. 
166 This study designed a sensory substitution device consisting of four electropiezo force sensors

167 (FlexiForce A401, Tekscan, Inc.) and two miniaturized vibrators. We instrumented an insole

168 with the force sensors at four critical locations, including the areas under the calcaneus

169 tuberosity, the fourth metatarsal, the first metatarsal, and the hallux (Figure 1A). One of our

170 previous researches has found that the force readings from these four locations can capture

171 most of the data variance in plantar pressure during walking (8). Since the feet size varied

172 among participants, we customized the shape of the insole for each individual participant. The

173 sensor was circular with a diameter of $2.54 \mathrm{~cm}$ and a thickness of merely $0.208 \mathrm{~mm}$. The

174 response time of the sensor was less than $5 \mu$ s with a sampling rate of $100 \mathrm{~Hz}$.

175 The vibrotactile feedback was delivered by the two circular vibrators, which were $12 \mathrm{~mm}$ in

176 diameter, $3.4 \mathrm{~mm}$ in height, and $1.7 \mathrm{~g}$ in mass. They were placed along the long axis of the

177 forearm of the affected side for amputees (Figure 1A). For the control participants, both the

178 instrumented insole and the vibrators were placed on the body's left side. The two vibrators

179 were separated by $10 \mathrm{~cm}$, which was distant enough to prevent possible perceptual ambiguity

180 across the simulated locations. The vibration amplitude and frequency were coupled together

181 for the miniaturized vibrators. Thus, we only adjusted their vibration intensity by a pulse

182 width modulation (PWM). The vibration intensity was modulated by the duty cycle of the

183 PWM signal. Both the force sensors and the vibrators were connected to a tablet computer

184 (Microsoft Surface 4) via an RS232 serial interface with a customized driver circuit. A

185 customized Matlab application was used for real-time signal processing (Mathworks, version 
A

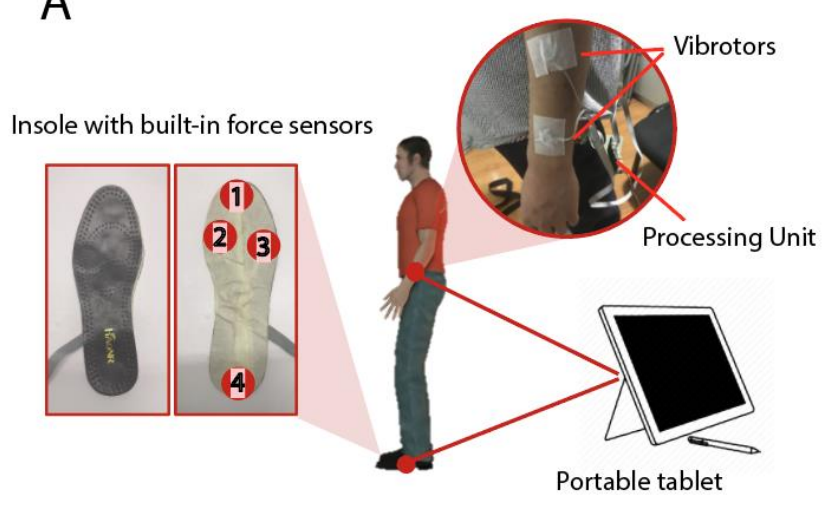

B

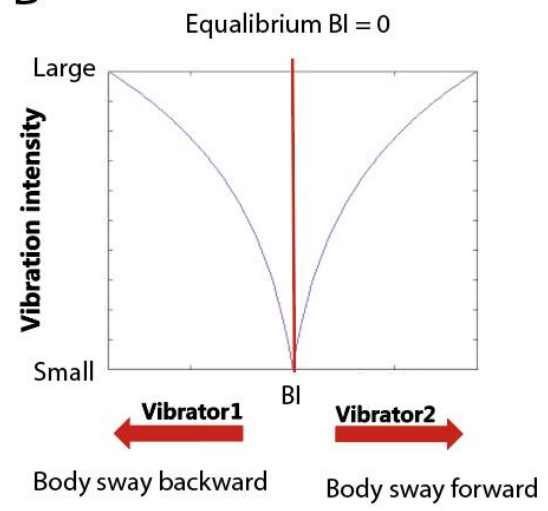

Figure 1: Illustration of the sensory substitution system. (A) The insole is instrumented with four thin electropiezo force sensors whose measurements are routed to a tablet for real-time data processing. The measured force determines the vibration intensity of the two tactile vibrators attached along the forearm's longitudinal axis on the same side of the insole. When participants walk or stand still, the vibration provides real-time feedback of the balance performance from the measured foot. (B) The vibration intensity follows a logarithmic relationship with the balance index (BI), which is determined by the changing force loading caused by body sway. Forward or backward body lean would lead to one vibrator working, respectively. 
203 (marked as 1 to 3 in Figure 1A) and the force of the $4^{\text {th }}$ force sensor placed under the hallux:

$$
B I=\frac{(F 1+F 2+F 3) / 3}{F 4}
$$

Where F1, F2, F3, and F4 are the readings from the four force sensors, respectively. Thus, the changes in the amplitude of $B I$ denote the postural sway in the anteroposterior direction. If the body leans forward, the signal strength of the force sensors in the forefoot will increase while the signal strength of the $4^{\text {th }}$ force sensor under the heel would decrease, increasing $B I$.

209 Conversely, a backward body sway would lead to a decrease in $B I$. We estimated the average

$210 B I$ for the neutral posture when each participant was asked to stand still without any

211 disturbance in so-called baseline trials (see below). This average $B I$ was defined as an

212 equilibrium point (EP), and typically the $B I$ would oscillate around each subject's EP. The $B I$

213 changes around the EP would be transformed into vibrotactile stimuli delivered to the

214 forearm.

215 To reduce the ambiguity of vibrotactile signals, we only activated one vibrator at a time:

216 when the $B I$ was larger than the EP, the vibrator placed in the front would vibrate to signal a

217 forward lean, and vice versa. The intensity of vibration for each vibrator was determined by

218 the absolute difference in $B I$ between the current state and the equilibrium state at EP:

$$
\text { Intensity }=\frac{\log \left(B I-B I_{E P}\right)}{\log B I_{\max }}
$$

Where $B I_{E P}$ is the average $B I$ estimated at $\mathrm{EP}$ when no visual perturbation was applied, and $B I_{\max }$ is the maximum $B I$ in the forward or the backward direction estimated from the trials 
when the participants first encountered visual perturbation on day 1 (sensory substitution was

223 off; see below). The relation between the vibration intensity and the $B I$ followed a logarithmic

224 function (Figure 1B). When the $B I$ slightly oscillated around the equilibrium point as

225 participants maintained a relatively neutral position, the vibrotactile feedback was weak. As

226 the $B I$ deviated more from EP, the intensity would increase, approaching the maximum

227 vibration intensity specified by the maximum $B I$ estimated in the baseline trials. Thus small

228 body sways would be more perceivable with the logarithmic transformation than a simple

229 linear function. Correspondingly, for large body sways, the tactile stimulation is not as strong

230 as with a linear function. We "sacrifice" the range of large signals in our tactile coding since

231 large body sways are readily perceivable by other sensory modality such as vision and

232 proprioception. Furthermore, studies of human psychophysics indicated that tactile perceptual

233 discrimination deteriorates with stimulus intensity $(35,36)$, suggesting that large tactile

234 signals are less informative. Thus, our sensory substitution's encoding scheme is to highlight

235 the feedback of small body sways but discount that of large body sways in keeping with

236 psychophysics principles.

\section{Participants}

238 We recruited seven transtibial amputees as the test group (including six males and one female

239 with an average age of $40.86 \pm 9.40$ years old) and eight non-disabled participants as the

240 control group (including six males and two females with an average age of $23.13 \pm 1.69$

241 years). The amputation time for amputee participants ranges from 8 to 26 years $(15.29 \pm 5.99$

242 years). Amputation was on the left side for six amputees and on the right for one amputee. All 
243 participants recruited in this study had no neuromotor disease or severe cardiovascular and

244 cerebrovascular diseases. All of them provided informed and written consent before the

245 experiment and were paid for their participation. The Institutional Review Board of Peking

246 University approved all procedures.

\section{Experiment}

248 The whole experiment was split into two parts and completed in two successive days. On day

249 1, all participants finished four blocks of 36 trials wearing the sensory substitution system

250 without turning on the vibration. However, their plantar pressure data were collected during

251 quiet standing. These trials also serve as baseline trials for computing $B I_{E P}$ and $B I_{\max }$. In this

252 way, we took the individual difference of body weight and foot conditions into consideration

253 for designing individualized vibrotactile stimulation for each participant. These parameters

254 were adopted in the sensory substitution system for subsequent tests of posture stability under

255 visual disturbances.

256 After baseline trials, postural stability was evaluated with the moving-room paradigm. The

257 experiment was conducted in a dark room while the participant maintained a quiet standing

258 posture $50 \mathrm{~cm}$ in front of a back-projection screen (Figure 2). The visual stimuli to provide

259 postural disturbance were projected onto the vertically-installed translucent screen by a

260 projector (InFocus, model IN104). The viewing area was $102 \mathrm{~cm}$ long and $68 \mathrm{~cm}$ high,

261 centered in between two eyes. Throughout the experiment, the participants wore a pair of

262 goggles limiting the field of view to approximately $120^{\circ}$ wide and $60^{\circ}$ high. Thus, the screen

263 edge was not visible to the participant, preventing it from being served as a visual reference 
264 for stabilizing posture. We tracked participants' head movements throughout the experiment

265 by an infrared motion capture system (OptiTrack, V120: Trio, Natural Point Inc.). A

266 reflective marker was fixed on the goggle side and approximately centered in the

267 measurement volume of the motion capture cameras. As participants stood on a plantar

268 pressure mat (RsScan Inc., Model footscan), their center of pressure (CoP) movements were

269 simultaneously measured along with their head movement. The sampling frequency was set at

$27060 \mathrm{~Hz}$ for both measurements. The stimuli presentation was generated by using Psychtoolbox

271 package in Matlab, and data acquisition was controlled by a single customized Matlab

272 program (Mathworks, version 2013a). We also used customized Matlab codes for data

273 analysis.

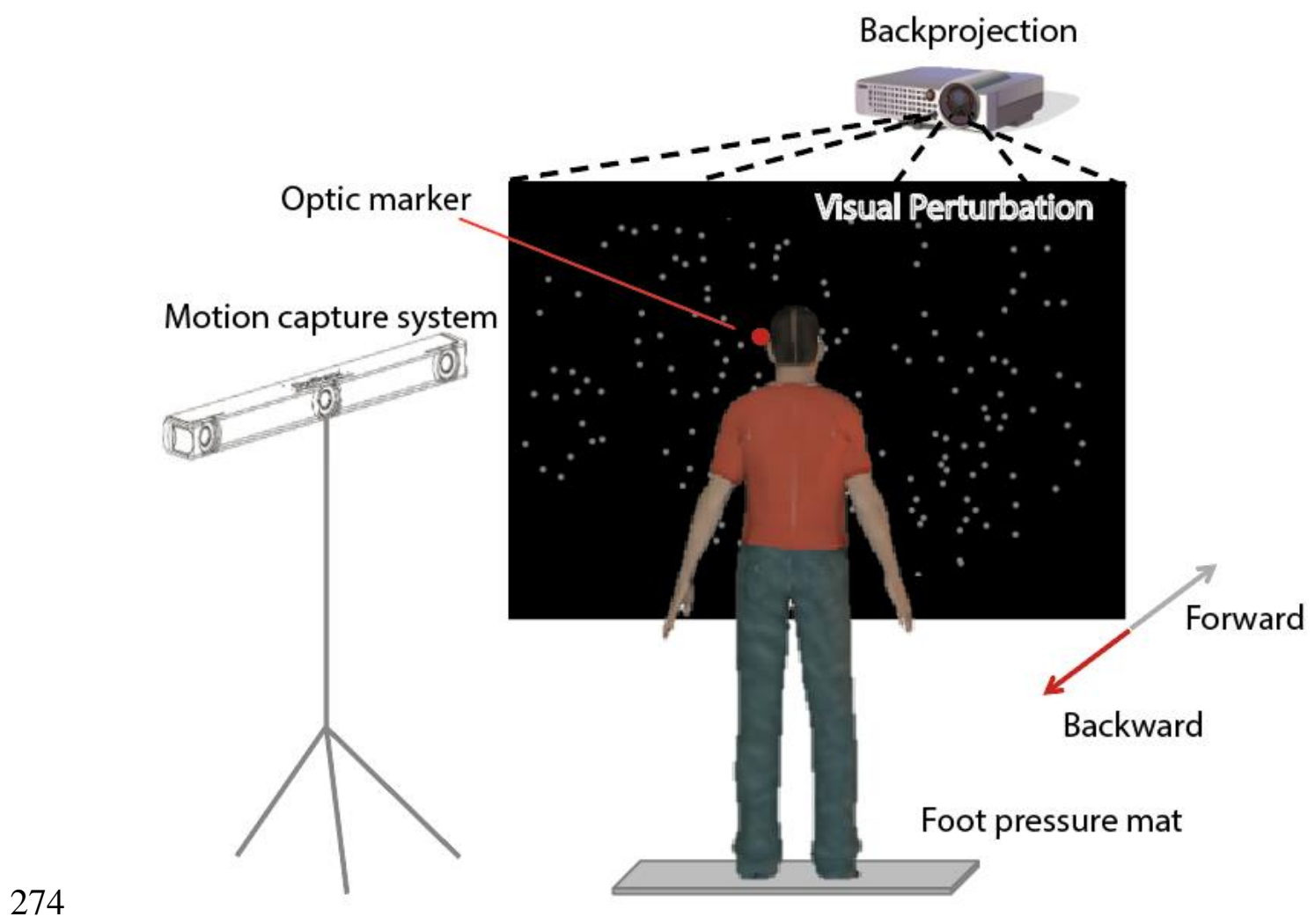


275 Figure 2: Illustration of the experimental setup for the postural stability test. The participant

276 stands on a plantar pressure mat, facing a large projection screen. The field of view is limited

277 to the screen by asking the participant to wear a pair of goggles. The head motion is

278 simultaneously tracked by a motion tracking system with a marker placed at the eye level.

279 The screen displays a cloud of random dots with simulated motion in the depth direction to

280 perturb the standing posture in the anteroposterior direction.

282 For the postural stability test, we adopted the classical moving-room paradigm where the

283 visual oscillatory disturbance was continuously presented to the participant $(42,43)$. The

284 stimulus consisted of 200 randomly generated dots, each with a size of 0.57 deg in diameter.

285 The dots were randomly distributed in an annulus between 10 deg and 45 deg visual

286 eccentricity (44). No stimulus was presented in the central foveal region to avoid aliasing

287 effects (42). Effectively, the dots simulated a space with depth before participants' eyes.

288 During the experiment, the depth of the visual scene oscillated in the anteroposterior

289 direction. This was achieved by changing the size of the dots and the distance between the

290 dots according to visual perspective. The anteroposterior movement of the visual stimulus

291 was sinusoidal with a certain frequency and amplitude. As the body sway was modulated by

292 both the frequency and amplitude of the oscillation, we used three frequencies $(0.1 / 0.3 / 0.5$

$293 \mathrm{~Hz})$ and three amplitudes $(2 / 4 / 8 \mathrm{~cm})$ to cover the parameter range typically reported in the

294 literature. This resulted in a total of nine stimulus conditions. 
295 Both the amputee group and the control group were examined for their postural stability with

296 and without sensory substitution. Each participant went through all the nine stimulus

297 conditions, four trials each condition. The total 72 trials were arranged as eight trial blocks,

298 four blocks with sensory substitution and the other four without. Each block thus consisted of

2999 trials, one trial for each of the nine stimulus conditions. Trials were randomly ordered

300 within each block. Each trial lasted 140s, and the first 20s were left out of subsequent analysis

301 since the large but transient postural sway at the beginning of a trial was a reflexive response

302 to the abruptly-induced visual disturbance (44). For testing their postural stability, participants

303 were instructed to fixate at the center of the display, which was left free of moving dots with a

$30410^{\circ}$ eccentricity. As the visual scene moved in the anteroposterior direction, the participant's

305 CoP was also displaced in the same direction, accompanied by $B I$ index changes and the

306 corresponding intensity changes of the tactile stimulation when the sensory substitution

307 system was on (Figure 3A). They were also encouraged to stand in a relaxed manner during

308 stimuli presentation. To prevent fatigue, we administrated a rest of 2 to 3 minutes between

309 trials and a mandatory rest of 5 minutes between blocks.

310 Given that the whole experiment lasted about 7 hours, we divided the whole experiment into

311 two days with four blocks of trials on each day. Participants needed to complete a total of 36

312 trials in 4 blocks without sensory substitution on day 1 to establish their baseline postural

313 stability before sensory substitution. They then completed another 4 blocks of 36 trials on day

3142 to examine the effect of sensory substitution. Note, as previous studies have not shown any 
315 habituation of visual disturbance in the moving room paradigm, we did not counterbalance

316 the conditions between days.

\section{Data analysis}

318 We analyzed the CoP or head movements while the participant was visually disturbed to

319 evaluate their postural stability (see Figure 3B for an exemplary trial). For each trial, we

320 computed the range of $\mathrm{CoP}$ and head movement in the A-P direction as a measure for postural

321 stability against the visual disturbance. The range measure specifically quantifies the

322 maximum body sway induced by the visual disturbance. We standardized the range of head

323 movement by dividing it with the height of each participant to minimize the effect of

324 individual differences in body height. We used Fourier transforms to analyze the CoP and

325 head movement in the anteroposterior direction and obtained the signal power over the

326 frequency range between 0 and $2 \mathrm{~Hz}$. As the frequencies of body sway and of visual

327 disturbance were way below $2 \mathrm{~Hz}$, this power measurement specifically quantifies the average

328 body sway over time. Thus, both range and power measurements quantify the postural

329 stability with their larger values corresponding to less stability. For each measure, we

330 conducted a 4-way mixed-design ANOVA with 3 (stimuli frequency) x 3 (stimuli amplitude)

331 x 2 (sensory substitution on vs. off) $\times 2$ (amputee vs. control group). As we observed the

332 stability improvement across all the conditions, we also computed the performance

333 improvement in the four performance variables by taking their difference between with and

334 without sensory substitution. Then, the improvements were submitted to 3-way mixed-design

335 ANOVA with 3 (stimuli frequency) x 3 (stimuli amplitude) x 2 (amputee vs. control group) to 


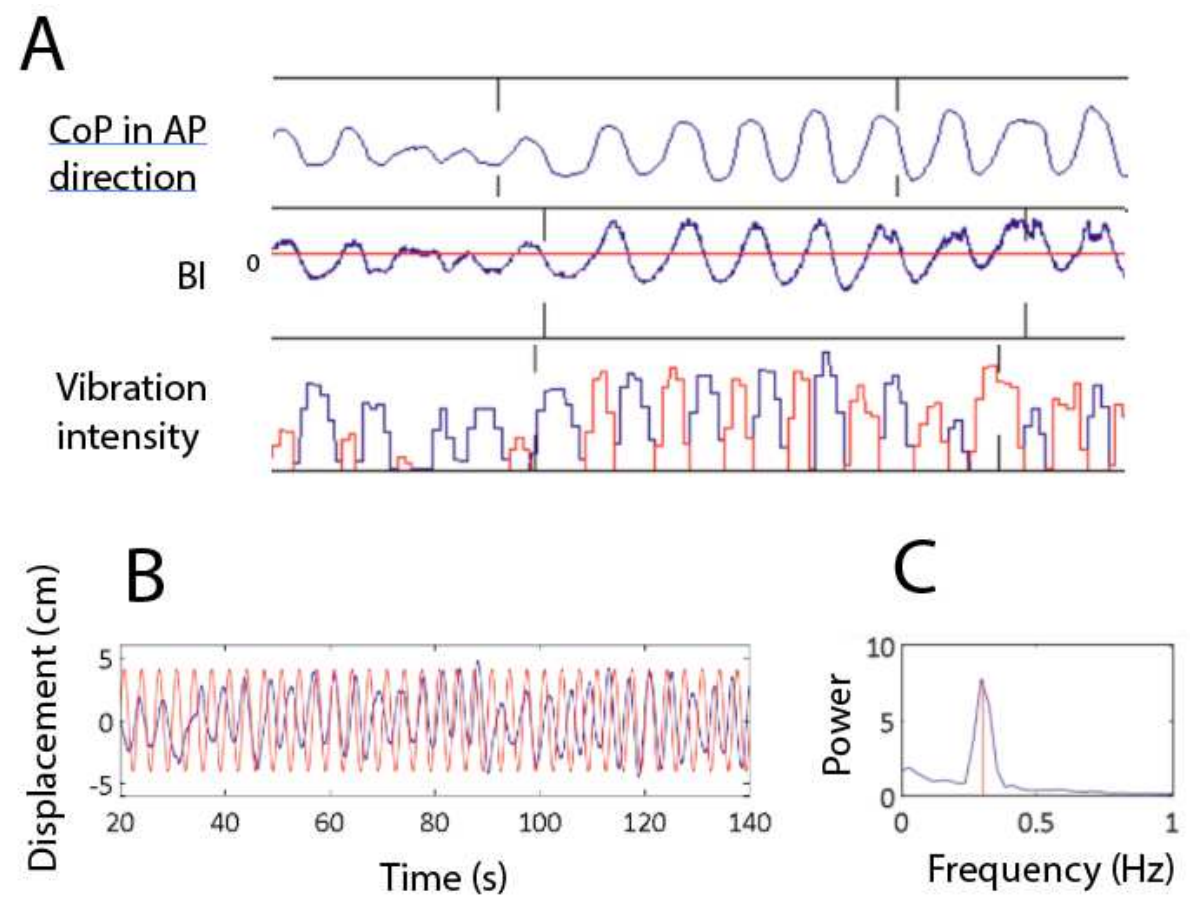

340 Figure 3: Exemplary data from the moving room paradigm. A) An exemplary trial segment to

341 show how the sensory substitution system works. The participant is perturbed by the

342 oscillatory visual stimuli, resulting in large CoP displacement in the anteroposterior (AP)

343 direction. Our system computed the $B I$ index in real-time and changed the vibration intensity

344 of the two vibrators (shown in blue and red, respectively) placed on the forearm of the

345 participant. B) An exemplary trial with head displacement (blue) and visual stimulus

346 displacement (red) in the anteroposterior direction. The formal data collection begins at the

$34720^{\text {th }}$ second. C) The power spectrum of head movement data of the same trial. The frequency 
348 of the visual stimulus here is $0.3 \mathrm{~Hz}$. The integral of the power over the frequency range

349 between 0 and $2 \mathrm{~Hz}$ was used for evaluating postural stability.

\section{$351 \quad$ Results}

352 We found that the visual disturbance modulated coP displacement and head movement, and

353 the body sway was reduced when the sensory substitution system was on for both groups of

354 participants. These effects can be readily shown as reduced CoP displacement (Figure 4 \& 5)

355 and head movement (Figure $6 \&$ 7). The four-way ANOVA on the range of CoP displacement

356 revealed no main effect of group $\left(F_{(1,13)}=0.12, p=0.74\right.$, partial $\left.\eta^{2}=0.009\right)$, but a

357 significant main effect of sensory substitution $\left(F_{(1,13)}=19.47, p=0.001\right.$, partial $\left.\eta^{2}=0.60\right)$.

358 Across groups, the CoP range before applying sensory substitution was larger than after (5.22

$359 \pm 0.77 \mathrm{~cm}$ v.s. $3.38 \pm 0.47 \mathrm{~cm}$, mean \pm std. error, same below). In fact, the improvement in

360 CoP stability was significant in all the nine stimulus conditions $(3$ stimulus frequency $\times 3$

361 amplitude) after Bonferroni correction (all $p s<0.01$ ). The main effect of stimulus frequency

362 was not significant $\left(F_{(2,26)}=1.48, p=0.26\right.$, partial $\left.\eta^{2}=0.10\right)$ but the main effect of the

363 stimulus amplitude was $\left(F_{(2,26)}=12.62, p<0.001\right.$, partial $\left.\eta^{2}=0.49\right)$. For interaction effect,

364 only the interaction between stimulus frequency and sensory substitution reached significance

$365\left(F_{(2,26)}=5.85, p=0.008\right.$, partial $\left.\eta^{2}=0.31\right)$. This interaction suggested that the benefit

366 brought by sensory substitution was larger in the conditions with a higher frequency, which

367 was more perturbing than lower frequency conditions. 


\section{Control}

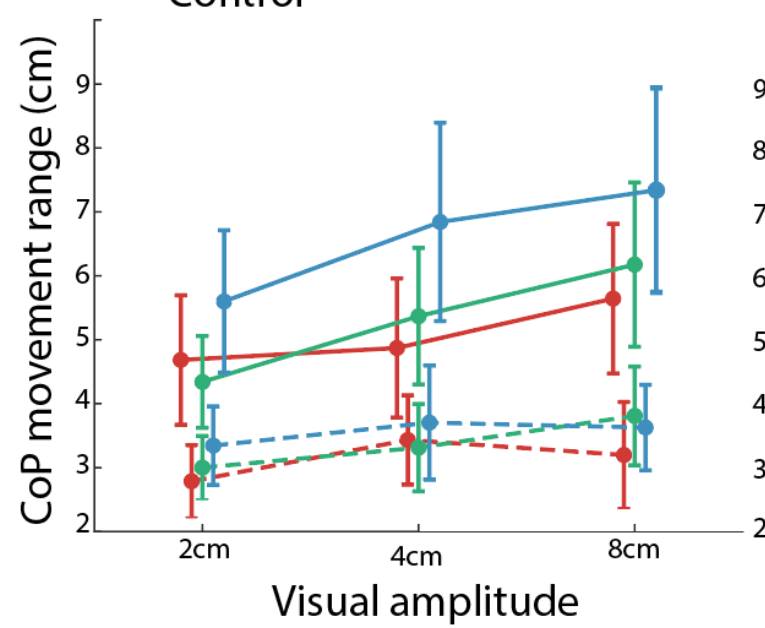

Amputee

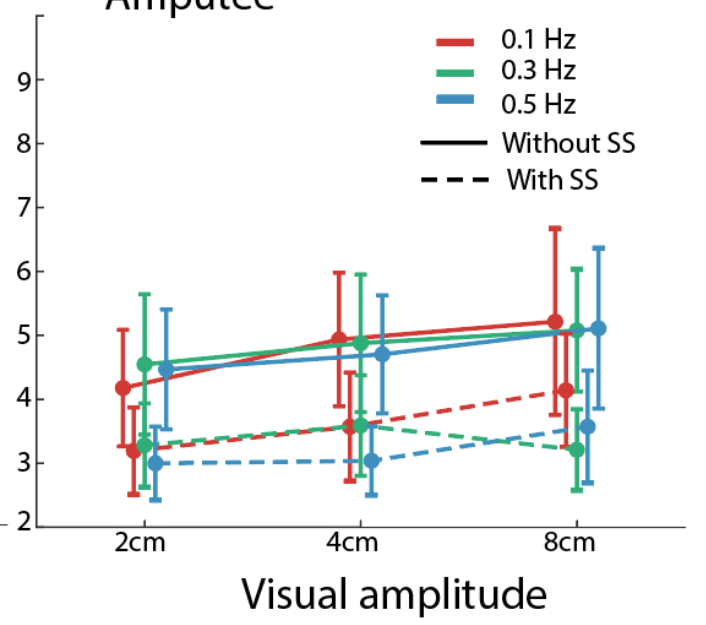

369 Figure 4: The range of $\mathrm{CoP}$ displacement in the anteroposterior direction plotted as a function

370 of stimulus amplitude and frequency. The conditions with and without sensory substitution

371 (SS) are shown in separate lines. The able-bodied control group and the amputee group are

372 shown in the left and right panels, respectively.

374 Power spectrum analysis of CoP displacement showed similar effects as the CoP range

375 (Figure 5). The power of CoP displacement was submitted to the same four-way ANOVA.

376 The main effect of sensory substitution was significant $\left(F_{(1,13)}=9.197, p=0.010\right.$, partial $\eta^{2}$

$377=0.41$ ), indicating that turning on the sensory substitution system reduced the COP excursion

378 in response to the visual disturbance. The main effects of group and stimulus frequency were

379 not significant $\left(F_{(1,13)}=1.40, p=0.26\right.$, partial $\eta^{2}=0.10$ for group; $F_{(1.19,15.47)}=2.00, p=$

3800.177 , partial $\eta^{2}=0.13$ for stimulus frequency). The main effect of stimulus amplitude was

381 marginally significant $\left(F_{(1.04,13.51)}=4.24, p=0.058\right.$, partial $\left.\eta^{2}=0.25\right)$. None of the

382 interaction effects was significant except the interaction between sensory substitution and 
383 stimulus amplitude $\left(F_{(1.06,13.74)}=4.59, p=0.049\right.$, partial $\left.\eta^{2}=0.26\right)$. The interaction, again,

384 indicates that the benefit of sensory substitution was more pronounced in the conditions with

385 larger visual amplitudes than with lower amplitudes. Overall, power spectrum analysis

386 revealed reduced body sway for both groups of participants when the sensory substitution

387 system was on.

388
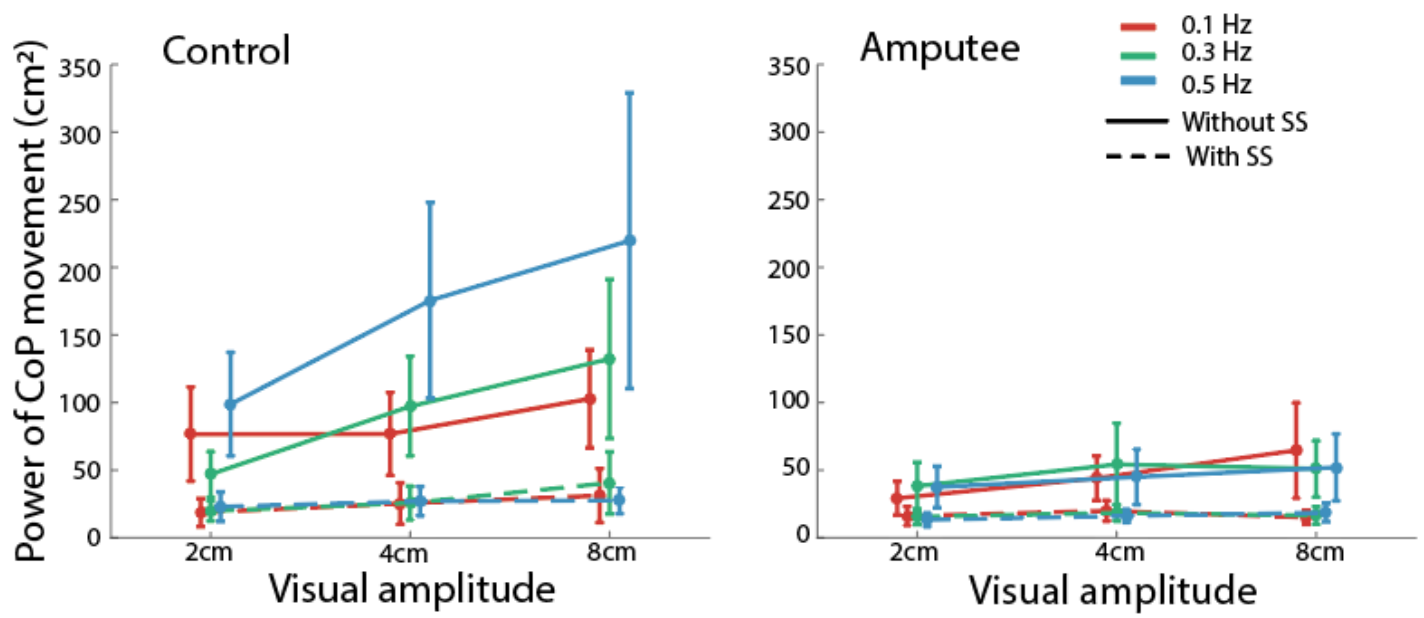

389 Figure 5: The power of CoP displacement plotted as a function of stimulus amplitude and

390 frequency. The conditions with and without sensory substitution (SS) are shown in separate

391 lines. The able-bodied control group and the amputee group are shown in the left and right

392 panels, respectively.

393

394 We further examined how the improvement of CoP sway by sensory substitution varied

395 across stimulus conditions. The reduction in CoP range by sensory substitution was computed

396 for each condition and then submitted to a 2 (group) $\times 3$ (stimulus frequency) $\times 3$ (stimulus

397 amplitude) mixed-design ANOVA. Either the main effect of group or stimulus amplitude was

398 significant $\left(F_{(1,13)}=1.17, p=0.298\right.$, partial $\eta^{2}=0.08$ for group; $F_{(2,26)}=2.42, p=0.109$, 
partial $\eta^{2}=0.16$ for stimulus amplitude). However, the main effect of stimulus frequency was significant $\left(F_{(2,26)}=5.85, p=0.008\right.$, partial $\left.\eta^{2}=0.31\right)$. None of the interactions was significant. The same ANOVA on the power of CoP displacement yielded similar results: no group difference or interaction was detected. However, the main effect of stimulus amplitude but not of stimulus frequency reached significance $\left(F_{(1.06,13.74)}=4.59, p=0.049\right.$, partial $\eta^{2}=$ 0.26 for stimulus amplitude; $F_{(1.15,14.91)}=1.67, p=0.219$, partial $\eta^{2}=0.11$ for stimulus frequency). These results suggest that both groups of participants benefited more from sensory substitution in those more challenging conditions with larger stimulus frequency or amplitude.

While the CoP displacement reflects the overall body weight shifts during standing, the head movement directly reflects the body sway at the eye level. We found that head movements

410 also showed a similar benefit of sensory substitution (Figure 6). For the head movement 411 range, the main effect of group was not significant $\left(F_{(1,13)}=0.05, p=0.820\right.$, partial $\eta^{2}=$

412 0.004). The average head movement range was comparable between the amputee group (4.20

$413 \pm 0.75 \mathrm{~cm})$ and the control group $(4.44 \pm 0.70 \mathrm{~cm})$. Importantly, the main effect of sensory

414 substitution was significant $\left(F_{(1,13)}=11.98, p=0.004\right.$, partial $\left.\eta^{2}=0.48\right)$. Across groups,

415 the head movement range decreased from $4.94 \pm 0.66 \mathrm{~cm}$ to $3.71 \pm 0.40 \mathrm{~cm}$ when the sensory

416 substitution was used. Again, we found that the improvement brought by sensory substitution

417 was significant in all nine stimulus conditions (all $p s<0.05$ after Bonferroni correction) except 418 in the condition with $0.3 \mathrm{~Hz}$ stimulus frequency and $2 \mathrm{~cm}$ amplitude (marginally significant 419 with $p=0.098)$. The main effect of stimulus frequency was not significant $\left(F_{(2,26)}=0.75, p=\right.$ 
4210.002 , partial $\eta^{2}=0.38$ ). Thus, stimulus amplitude, but not stimulus frequency, modulated 422 the head motion, a similar result as the CoP range. All the interactions failed to reach

423 significance except the interaction between sensory substitution and stimulus amplitude $\left(F_{(2,}\right.$

$424{ }_{26)}=3.74, p=0.037$, partial $\eta^{2}=0.22$ ), again suggesting that the benefit of sensory

425 substitution was more pronounced with larger visual disturbances.
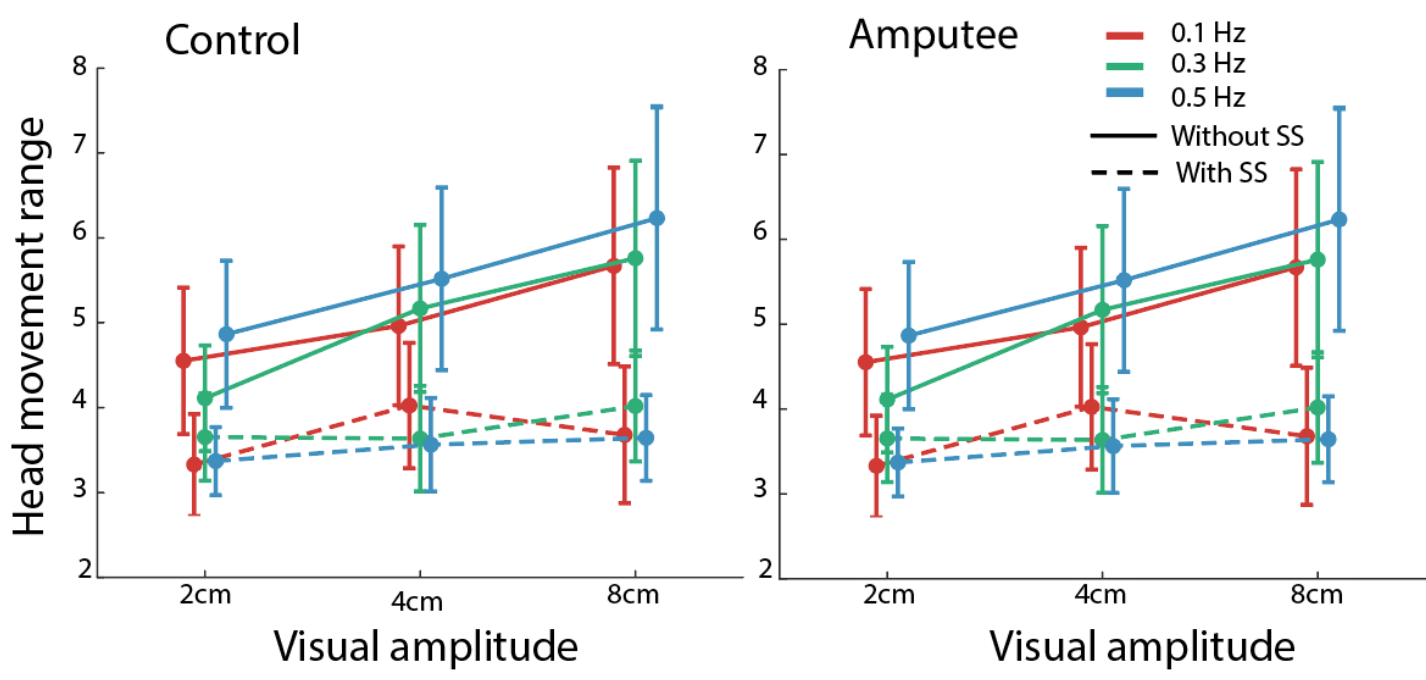

427 Figure 6: The head movement range in the anteroposterior direction plotted as a function of

428 stimulus amplitude and frequency. The conditions with and without sensory substitution (SS)

429 are shown in separate lines. The able-bodied control group and the amputee group are shown

430 in the left and right panels, respectively. Note the head movement range is unit-less as it is

431 normalized by dividing the participant's body height.

433 Power spectrum analysis of head movement revealed a similar pattern as the range of head

434 movement (Figure 7). The power of head movement was submitted to the same four-way 
ANOVA. The main effect of group was not significant $\left(F_{(1,13)}=1.40, p=0.259\right.$, partial $\eta^{2}$

$436=0.10)$. The main effect of sensory substitution was significant $\left(F_{(1,13)}=9.20, p=0.010\right.$,

437 partial $\eta^{2}=0.41$ ), and the power decreased when the sensory substitution system was on.

438 Main effect of stimulus amplitude was marginally significant $\left(F_{(1.04,13.51)}=4.24, p=0.058\right.$,

439 partial $\left.\eta^{2}=0.25\right)$, but not for stimulus frequency $\left(F_{(1.19,15.47)}=2.00, p=0.177\right.$, partial $\eta^{2}=$

440 0.13). None of the interactions was significant except the interaction between sensory

441 substitution and stimulus amplitude $\left(F_{(1.17,15.22)}=5.53, p=0.028\right.$, partial $\left.\eta^{2}=0.30\right)$. Thus,

442 while the larger stimulus amplitude tended to cause larger body sway, the same sensory

443 substitution effect was also larger with larger stimulus amplitudes.

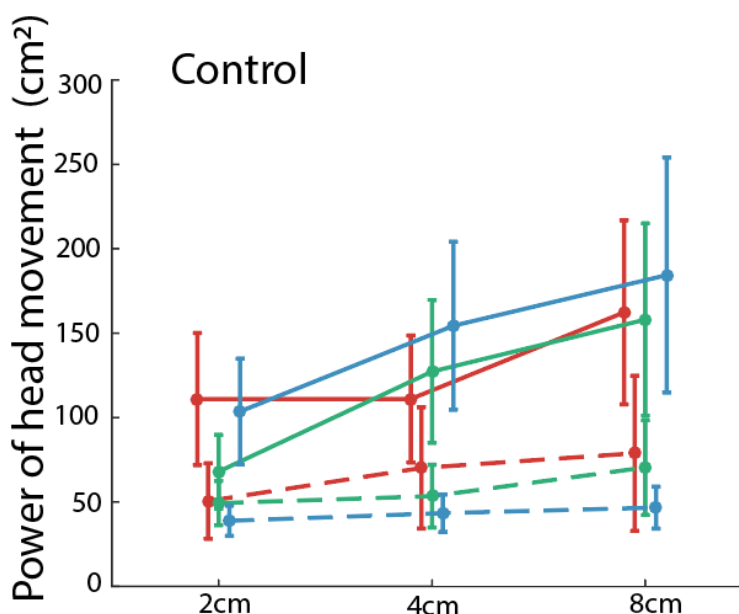

Visual amplitude

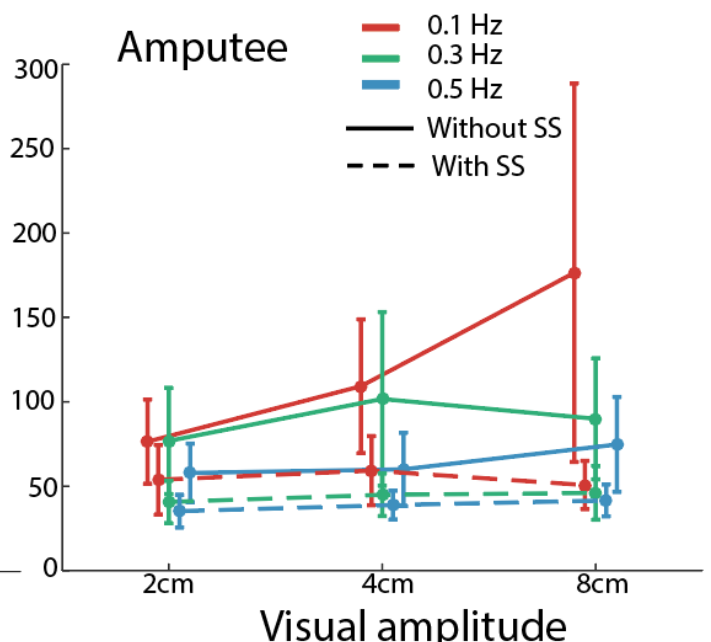

Visual amplitude

445 Figure 7: The power of head movement plotted as a function of stimulus amplitude and

446 frequency. The conditions with and without sensory substitution (SS) are shown in separate

447 lines. The able-bodied control group and the amputee group are shown in the left and right 448 panels, respectively. 
We then examined how the improvement in head stabilization brought by sensory substitution partial $\eta^{2}=0.05$ and $F_{(2,26)}=0.86, p=0.436$, partial $\eta^{2}=0.06$, respectively). None of the interactions was significant. The reduction in the power of the head movement was also examined by the same three-way ANOVA. Again, no main effect of group or interaction was detected. The main effect of stimulus amplitude but not stimulus frequency was significant $\left(F_{(1.17,15.22)}=5.53, p=0.028\right.$, partial $\eta^{2}=0.30$ and $F_{(1.26,16.44)}=0.22, p=0.698$, partial $\eta^{2}=$ conditions.

\section{Discussion}

464 This study aims to investigate whether lower-limb amputees can improve their postural 
471 sensory substitution was applied across various visual conditions. We also found that the

472 balance improvement brought by sensory substitution was more pronounced for more

473 challenging conditions with larger visual disturbance. Thus, our findings suggest that closing

474 the broken sensorimotor loop of lower-limb amputees by using real-time sensory substitution

475 can help improve postural control and, potentially, other actions that involve ground-foot

476 interactions.

477 Postural control is under the simultaneous influence of multiple sensory modalities, including

478 visual, vestibular, proprioceptive, and tactile modalities. For maintaining postural stability

479 during standing, the nervous system adjusts the relative contributions of sensory inputs from

480 different channels during the multisensory integration process according to sensory precision

481 of individual channels (45-47). In the moving-room paradigm, the visual scene oscillates and

482 biases the estimated standing posture, resulting in postural sway (44). Sensory inputs from

483 other modalities, including the augmented tactile feedback applied on body parts other than

484 the foot, can negate the visual disturbance. For example, researchers have shown that the light

485 touch of a fingertip on a stable surface can provide subtle tactile feedback for stabilizing

486 posture during quiet standing and standing under visual interference $(48,49)$. Vuillerme,

487 Chenu (50) used a $6 \times 6$ electrotactile matrix on the tongue to provide feedback of CoP

488 changes for the non-disabled participants and improve their postural stability in a condition

489 where neck proprioceptive and vestibular inputs were compromised with an unnatural

490 posture. Thus, extra tactile inputs can indeed improve postural stability among non-disabled

491 participants. Our study went a step further to show that lower-limb amputees could improve 
493 the plantar pressure changes. Presumably, this stabilizing effect follows the same sensory

494 integration principles that have been repeatedly reported in different paradigms (51).

495 Previous studies using similar vibrotactile feedback to substitute foot pressure have failed to

496 show consistent benefit in postural stability (e.g., 26). We postulate that differences in the

497 tactile coding scheme and the postural test are responsible for the discrepancy. The

498 intuitiveness and comfort of augmented tactile feedback presented to the human wearer were

499 not systematically investigated until recently (52). Our approach paid particular attention to

500 make the tactile feedback intuitive. First, only bodyweight shifts, as measured by plantar

501 pressure underneath the foot, were encoded. This is in contrast to the one-to-one signal

502 mapping between a pressure sensor and a tactor in previous studies (e.g., 26). One-to-one

503 mapping is technically straightforward, but it would pose a challenge for the wearer to

504 understand tactile signals' meaning. Second, our system encodes the body sway in the

505 anteroposterior direction, the prominent direction of instability during quiet standing, with the

506 two tactile stimulators aligned with the forearm's longitudinal axis. Thus, the tactile feedback

507 is spatially congruent with the visual disturbance and postural sway. Third, we limited the two

508 stimulators to work one at a time and used a logarithmic transfer function to use better the

509 perceptual range of tactile stimuli $(35,36)$. These signal designs help resolve the so-called

510 neutral zone problem when people receive little tactile feedback around a neutral posture (26).

511 These design aspects appeared to help participants, especially amputee participants who have 
512 not received direct foot contact pressure information for long, quickly learn to use surrogate

513 sensory feedback to improve their postural control.

514 It is noteworthy that the benefit of our sensory substitution system manifested itself without

515 extensive training. Our participants familiarized themselves with the system on day one over

51636 trials. Previous studies on sensory substitution typically required several weeks of practice

517 time $(53,54)$. We postulate that the intuitive encoding scheme of the vibrotactile feedback

518 facilitated this fast adoption of sensory substitution.

519 We used three stimulus frequencies $(0.1 / 0.3 / 0.5 \mathrm{~Hz})$ and three amplitudes $(2 / 4 / 8 \mathrm{~cm})$ to

520 perturb the participant visually in our postural control task. We found that the amplitude of

521 visual stimulus predominantly affected postural stability, as shown by different independent

522 measures. When the amplitude of visual motion increased, the body sway increased, as shown

523 in head movements and overall CoP displacement, consistent with previous research (55).

524 The oscillation frequency of visual disturbance showed an inconsistent effect on body sway.

525 For example, control participants tend to increase their power of $\mathrm{CoP}$ displacement and head

526 movement with increasing stimulus frequency, but amputee participants showed an opposite

527 tendency (Figure 5 and 7). When visual stimuli moved with a lower frequency (e.g., $0.1 \mathrm{~Hz}$ ),

528 the body swayed periodically in synchrony with the driving visual stimuli. When visual

529 stimuli moved with a high frequency (e.g., $0.5 \mathrm{~Hz}$ ), it became hard for the body sway to keep

530 up with the stimuli, resulting in a smaller power $(42,56)$. This saturation effect appears to be

531 more evident for amputees than for non-disabled participants. 
532 We also computed the performance difference before and after sensory substitution to

533 compare the effect size of sensory substitution across conditions. Three out of the four

534 measures (i.e., the power of CoP displacement, the range and the power of head movement)

535 showed a larger effect size in conditions with larger visual-stimuli amplitudes. The range of

536 CoP displacement, the last measure, did not increase with visual amplitude, but it did increase

537 with visual frequency. Thus, the sensory substitution system benefited both groups of

538 participants more when they were faced with more challenging visual disturbance.

539 We found that sensory substitution stabilized the head and CoP with similar effect sizes. For

540 the CoP range, the effect size of sensory substitution was 0.60 in partial $\eta^{2}$, which is

541 equivalent to a $35.3 \%$ reduction after sensory substitution. In comparison, for the head

542 movement range, the effect size was 0.48 with a $24.8 \%$ reduction. The same pattern was

543 found in the power analysis, where both CoP displacement and head movement yielded an

544 effect size of 0.41 with a reduction of $22.6 \%$. Previous research has established that humans

545 prioritize stabilizing the head with visual feedback when the overall posture changes (57).

546 Furthermore, if we assume that the standing body resembles an inverted pendulum as in

547 typical postural models (58), the head movement should decrease more when the CoP

548 decreases. Thus, theoretically, we shall expect a more significant stabilizing effect of sensory

549 substitution for the head than for the CoP. The lack of difference between the head and the

$550 \mathrm{CoP}$, or even a slightly more significant effect for the CoP, does not fit the theoretical

551 prediction. We postulate that this might be attributed to the specificity of surrogated sensory

552 information delivered by our sensory substitution system: the vibrotactile feedback reflects 
553 plantar pressure changes directly related to CoP excursion, not to head movement. Thus,

554 when the nervous system integrates this surrogate sensory information, it readily responds to

555 CoP displacement induced by visual disturbances. Therefore, our findings appear to suggest

556 that sensory substitution exerts its influence on motor control in a stimulus-specific way, at

557 least for the situation investigated here where sensory substitution is adopted for a short

558 period of time. Future studies could test this hypothesis by comparing the responses to

559 substituted stimuli that encoded different body motion signals, e.g., head motion instead of

560 CoP displacement.

561 Interestingly, no group difference of postural stability between amputees and the control

562 reached significance for all the performance measures investigated. We expected that

563 amputees would be perturbed more by the visual disturbances since previous studies have

564 shown that amputees are more dependent on visual inputs (39-41). However, we recognize

565 that these studies used paradigms that reduced visual sensory feedback for the participants.

566 Understandably, it was harder for amputees than the non-disabled to accommodate visual

567 deprivation due to the loss in somatosensory feedback associated with amputation. In the

568 present study, however, we used a visual perturbation paradigm rather than visual deprivation.

569 According to multisensory integration theory in postural control (45-47), both amputees and

570 the non-disabled could adjust the weights of different sensory channels when sensory inputs

571 (i.e., visual input) became inaccurate. Furthermore, previous studies reported worse standing

572 balance among amputees typically used short trials, e.g., 20 s per trial (59). Our experiment

573 instead used as long as $140 \mathrm{~s}$ per trial; thus, both groups had ample time to adjust their 
574 weights of different sensory channels and adapt to the visual stimuli. The other factor is that

575 most of our participants have worn artificial limbs for more than ten years. After prolonged

576 use of prosthesis, their performance in simple motor tasks such as quiet standing become

577 indistinguishable from that of the non-disabled. In sum, the lack of group difference thus

578 suggests that lower-limb amputees can effectively accommodate continuous visual

579 disturbances.

580 The development of robotic artificial limbs has been made dramatic progress in fusing signals

581 from various sensors for sensing the environment and the internal state of the prosthesis, but

582 the research focus is more on intelligent control of prostheses (60). It is equally essential to

583 route real-time sensory feedback for the agent, i.e., the human controller, to reduce the fear of

584 falling, enhance the sense of embodiment of the prosthesis, and better motor control. This

585 sensory augmentation for the agent can be achieved by invasive methods such as electrical

586 peripheral nerve stimulation of the sciatic nerve (61) or noninvasive methods such as sensory

587 substitution. As we pointed out in the introduction, substituting the missing feedback of foot-

588 ground interaction is probably most important for lower-limb amputees. Still, the previous

589 endeavors have been hampered by high demands of cognitive loads, unintuitive design, and

590 inconsistent behavioral benefits. Our study has shown that these shortcomings of noninvasive

591 sensory substitution can be overcome. It paves the way for us to integrate this method with

592 robotic lower limbs. As most actuated lower-limb prostheses still lack afferent feedback to the

593 user, it would be interesting to examine the outcome when our sensory substitution system

594 integrates with these systems to achieve better human-centered close-loop control. 
595 Furthermore, even though our postural tests showed the feasibility and effectiveness of tactile

596 sensory substitution in stabilizing people's standing posture with minimal training, they were

597 limited to transtibial amputees in the laboratory environment with a classical experimental

598 task. Future endeavors should be directed to testing the system among transfemoral amputees

599 and via dynamic balancing tasks, such as walking on different surfaces. We expect the need

600 for specific modifications of the signal encoding scheme for diverse movement scenarios.

601

602 Conclusions

603 Using vibrotactile stimulation to substitute the missing plantar pressure information for

604 transtibial amputee leads to improvements in postural stability during visually-perturbed quiet

605 standing. Both amputees and able-bodied can benefit from sensory substitution, especially

606 when large visual perturbations challenge their posture. Future development for sensory

607 substitution shall consider making surrogated sensory inputs spatially congruent with the to-

608 be-simulated sensory inputs and following psychophysical principles.

609

610 List of abbreviations

611 CoP: center of pressure; BI: balance index; SS: sensory substitution; ANOVA: analysis of

612 variance

613 


\section{Declarations}

615

616

618

619

620

621

624

625

626

\section{- Ethics approval and consent to participate}

The study was designed following the Declaration of Helsinki. The study protocol (NSFC2018-06-02) was approved by the Ethical Committee of Peking University (Beijing, China). All participants gave written informed consent.

\section{- Consent for publication}

Not applicable

- Availability of data and materials

The datasets used during the current study are available from the corresponding author on reasonable request.

\section{- Competing interests}

None of the authors have any competing interests to report.

\section{- Funding}

This work was supported by the National Natural Science Foundation of China $(31671168,32071047,61533001,51922015,62061136001)$.

Authors' contributions 

performed by L.C., Y.F., and B.C.

\section{- Acknowledgments}

635

- The authors would like to thank Dr. Kebin Yuan for helping to recruit our

636 participants.

637

638

\section{References}

639 1. Wolpert DM, Ghahramani Z. Computational principles of movement neuroscience.

640 Nature neuroscience. 2000;3(11):1212-7.

641 2. Windrich M, Grimmer M, Christ 0, Rinderknecht S, Beckerle P. Active lower limb

642 prosthetics: a systematic review of design issues and solutions. Biomedical

643 engineering online. 2016;15(3):5-19.

644 3. Au SK, Weber J, Herr H. Powered Ankle--Foot Prosthesis Improves Walking

645 Metabolic Economy. IEEE Transactions on Robotics. 2009;25(1):51-66.

646 4. Hitt J, Sugar T, Holgate M, Bellman R, Hollander K. Robotic transtibial

647 prosthesis with biomechanical energy regeneration. Industrial Robot: An

648 International Journal. 2009;36(5):441-7. 
649 5. Lawson BE, Mitchell J, Truex D, Shultz A, Ledoux E, Goldfarb M. A robotic leg

650 prosthesis: design, control, and implementation. IEEE Robotics \& Automation

651 Magazine. 2014;21(4):70-81.

652 6. Sup F, Bohara A, Goldfarb M. Design and control of a powered transfemoral

653 prosthesis. The International journal of robotics research. 2008;27(2):263-73.

654 7. Wang Q, Yuan K, Zhu J, Wang L. Walk the walk: A lightweight active transtibial

655 prosthesis. IEEE Robotics \& Automation Magazine. 2015;22(4) :80-9.

656 8. Chen B, Wang Q, Wang L. Adaptive slope walking with a robotic transtibial

657 prosthesis based on volitional EMG control. IEEE/ASME Transactions on mechatronics.

$6582015 ; 20(5): 2146-57$.

659 9. Ha KH, Varol HA, Goldfarb M. Volitional control of a prosthetic knee using

660 surface electromyography. IEEE Transactions on Biomedical Engineering.

$6612011 ; 58(1): 144-51$.

662 10. Hoover CD, Fulk GD, Fite KB. Stair ascent with a powered transfemoral

663 prosthesis under direct myoelectric control. IEEE/ASME Transactions on

664 Mechatronics. 2013;18(3):1191-200.

665 11. Perry SD, McIlroy WE, Maki BE. The role of plantar cutaneous mechanoreceptors

666 in the control of compensatory stepping reactions evoked by unpredictable, multi-

667 directional perturbation. Brain research. 2000;877 (2):401-6. 
668 12. Miller WC, Speechley M, Deathe B. The prevalence and risk factors of falling

669 and fear of falling among lower extremity amputees. Archives of physical medicine

670 and rehabilitation. $2001 ; 82(8): 1031-7$.

671 13. Hunter SW, Batchelor F, Hill KD, Hill A-M, Mackintosh S, Payne M. Risk factors

672 for falls in people with a lower limb amputation: a systematic review. Pm\&r.

$6732017 ; 9(2): 170-80 . \quad$ e1.

674 14. Escamilla-Nunez R, Michelini A, Andrysek J. Biofeedback systems for gait

675 rehabilitation of individuals with lower-limb amputation: a systematic review.

676 Sensors. 2020;20(6):1628.

677 15. Ma CZ-H, Wong DW-C, Lam WK, Wan AH-P, Lee WC-C. Balance improvement effects of

678 biofeedback systems with state-of-the-art wearable sensors: A systematic review.

679 Sensors. $2016 ; 16(4): 434$.

680 16. Bach-y-Rita P, Kercel SW. Sensory substitution and the human-machine

681 interface. Trends in cognitive sciences. 2003;7(12):541-6.

682 17. Antfolk C, D’ alonzo M, Rosén B, Lundborg G, Sebelius F, Cipriani C. Sensory

683 feedback in upper limb prosthetics. Expert review of medical devices.

$6842013 ; 10(1): 45-54$.

685 18. Zambarbieri D, Schmid M, Magnaghi M, Vermi G, Macellari V, Fadda A.

686 Biofeedback techniques for rehabilitation of the lower-limb prosthetic subject.

687 Proc VII Medicon Lemesos, Cyprus: MEDICON. 1998:1-5. 
689 ambulation feedback system for analysis of gait asymmetries: preliminary design and

690 validation results. JP0: Journal of Prosthetics and Orthotics. 2010;22(1):31-6.

691 20. Yang L, Dyer P, Carson R, Webster J, Foreman KB, Bamberg S. Utilization of a amputation gait. Gait \& posture. $2012 ; 36(3): 631-4$.

694 21. Sabolich JA, Ortega GM. Sense of feel for lower-limb amputees: a phase-one

695 study. JP0: Journal of Prosthetics and Orthotics. 1994;6(2):36-41.

696 22. Buma DG, Buitenweg JR, Veltink PH. Intermittent stimulation delays adaptation

697 to electrocutaneous sensory feedback. IEEE Transactions on Neural Systems and

698 Rehabilitation Engineering. 2007;15(3):435-41.

699 23. Crea S, Cipriani C, Donati M, Carrozza MC, Vitiello N. Providing time-discrete 700 gait information by wearable feedback apparatus for lower-limb amputees: usability 701 and functional validation. IEEE Transactions on Neural Systems and Rehabilitation

702 Engineering. 2014;23(2):250-7.

703 24. Fan RE, Culjat M0, King C-H, Franco ML, Boryk R, Bisley JW, et al. A haptic

704 feedback system for lower-1imb prostheses. IEEE Transactions on Neural Systems and

705 Rehabilitation Engineering. 2008;16(3):270-7.

706 25. Fan RE, Wottawa C, Mulgaonkar A, Boryk RJ, Sander TC, Wyatt MP, et al., 707 editors. Pilot testing of a haptic feedback rehabilitation system on a lower-limb 

IEEE. 26. Rusaw D, Hagberg K, Nolan L, Ramstrand N. Can vibratory feedback be used to

711 improve postural stability in persons with transtibial limb loss? Journal of rehabilitation research and development. 2012;49(8):1239-54.

715 Annual International Conference of the IEEE Engineering in Medicine and Biology

716 Society; 2011: IEEE.

717 28. Kaczmarek KA, Webster JG, Bach-y-Rita P, Tompkins WJ. Electrotactile and

718 vibrotactile displays for sensory substitution systems. IEEE transactions on

719 biomedical engineering. 1991;38(1):1-16.

720 29. Plauché A, Villarreal D, Gregg RD. A haptic feedback system for phase-based

721 sensory restoration in above-knee prosthetic leg users. IEEE transactions on

722 haptics. $2016 ; 9(3): 421-6$.

723 30. Charkhkar H, Christie BP, Triolo RJ. Sensory neuroprosthesis improves postural

724 stability during Sensory Organization Test in lower-limb amputees. Scientific

725 Reports. 2020;10(1):1-13.

726 31. Petrini FM, Valle G, Bumbasirevic M, Barberi F, Bortolotti D, Cvancara P, et

727 al. Enhancing functional abilities and cognitive integration of the lower limb

728 prosthesis. Science translational medicine. 2019;11(512). 
730 vibrotactile balance prosthesis. Journal of Vestibular Research. 2006;16 (1, 2):45-

73156.

732 33. Goebel JA, Sinks BC, Parker Jr BE, Richardson NT, Olowin AB, Cholewiak RW.

733 Effectiveness of head-mounted vibrotactile stimulation in subjects with bilateral

734 vestibular loss: a phase 1 clinical trial. 0tology \& Neurotology. 2009;30 (2):210-6.

735 34. Sienko KH, Balkwill MD, Oddsson L, Wall C. Effects of multi-directional

736 vibrotactile feedback on vestibular-deficient postural performance during

737 continuous multi-directional support surface perturbations. Journal of Vestibular

738 Research. 2008;18 (5, 6) :273-85.

739 35. Craig JC. Difference threshold for intensity of tactile stimuli. Perception \&

$740 \quad$ Psychophysics. 1972;11(2):150-2.

741 36. Gescheider GA, Bolanowski Jr SJ, Verrillo RT, Arpajian DJ, Ryan TF.

742 Vibrotactile intensity discrimination measured by three methods. The Journal of the

743 Acoustical Society of America. 1990;87 (1):330-8.

744 37. Chen B, Feng Y, Wang Q. Combining vibrotactile feedback with volitional

745 myoelectric control for robotic transtibial prostheses. Frontiers in neurorobotics.

$746 \quad 2016 ; 10: 8$.

747 38. Vrieling A, Van Keeken H, Schoppen T, Otten E, Hof A, Halbertsma J, et al.

748 Balance control on a moving platform in unilateral lower limb amputees. Gait \&

749 posture. $2008 ; 28(2): 222-8$. 
39. Dornan J, Fernie GR, Holliday PJ. Visual input: its importance in the control

751 of postural sway. Archives of physical Medicine and Rehabilitation.

$7521978 ; 59(12): 586-91$.

753 40. Fernie GR, Holliday PJ. Postural sway in amputees and normal subjects. The

754 Journal of bone and joint surgery American volume. 1978;60(7):895-8.

755 41. Vanicek N, Strike S, McNaughton L, Polman R. Postural responses to dynamic perturbations in amputee fallers versus nonfallers: a comparative study with ablebodied subjects. Archives of physical medicine and rehabilitation. 2009;90(6) :1018-

75825.

759 42. Dijkstra T, Schöner G, Gielen C. Temporal stability of the action-perception

760 cycle for postural control in a moving visual environment. Experimental Brain

$761 \quad$ Research. 1994;97 (3):477-86.

762 43. Dijkstra T, Schöner G, Giese MA, Gielen C. Frequency dependence of the action-

763 perception cycle for postural control in a moving visual environment: relative

764 phase dynamics. Biological Cybernetics. 1994;71(6):489-501.

765 44. Wei K, Stevenson I, Körding K. The uncertainty associated with visual flow

766 fields and their influence on postural sway: Weber's law suffices to explain the

767 nonlinearity of vection. Journal of Vision. 2010;10(14).

768 45. Mergner T, Maurer C, Peterka R. A multisensory posture control model of human

769 upright stance. Progress in brain research. 142: Elsevier; 2003. p. 189-201. 
771 neurophysiology. 2002;88(3):1097-118.

772 47. Peterka RJ, Loughlin PJ. Dynamic regulation of sensorimotor integration in

773 human postural control. Journal of neurophysiology. 2004;91 (1) :410-23.

774 48. Jeka J, Oie KS, Kiemel T. Multisensory information for human postural control:

775 integrating touch and vision. Experimental Brain Research. 2000;134(1):107-25.

776 49. Jeka J, Schöner G, Dijkstra T, Ribeiro P, Lackner JR. Coupling of fingertip

777 somatosensory information to head and body sway. Experimental Brain Research.

$778 \quad 1997 ; 113(3): 475-83$.

779 50. Vuillerme N, Chenu 0, Pinsault N, Fleury A, Demongeot J, Payan Y. Can a

780 plantar pressure - based tongue-placed electrotactile biofeedback improve postural

781 control under altered vestibular and neck proprioceptive conditions? Neuroscience.

$7822008 ; 155(1): 291-6$.

783 51. Mergner T, Maurer C, Peterka R. A multisensory posture control model of human

784 upright stance. Progress in brain research. 2003;142:189-201.

785 52. Cesini I, Spigler G, Prasanna S, D' abbraccio J, De Luca D, Dell' Agnello F,

786 et al. Assessment of intuitiveness and comfort of wearable haptic feedback

787 strategies for assisting level and stair walking. Electronics. 2020;9(10):1676.

788 53. Bach-y-Rita P, Collins CC, Saunders FA, White B, Scadden L. Vision

789 substitution by tactile image projection. Nature. 1969;221(5184):963. 
790 54. Striem-Amit E, Guendelman M, Amedi A. 'Visual' acuity of the congenitally

791 blind using visual-to-auditory sensory substitution. PloS one. 2012;7(3):e33136.

792 55. Peterka R, Benolken M. Role of somatosensory and vestibular cues in

793 attenuating visually induced human postural sway. Experimental Brain Research.

$7941995 ; 105(1): 101-10$.

795 56. Mergner T, Schweigart G, Maurer C, Blümle A. Human postural responses to

796 motion of real and virtual visual environments under different support base

797 conditions. Experimental Brain Research. 2005;167 (4):535-56.

798 57. Guitton D, Kearney R, Wereley N, Peterson B. Visual, vestibular and voluntary

799 contributions to human head stabilization. Experimental brain research.

$8001986 ; 64(1): 59-69$.

801 58. Gage WH, Winter DA, Frank JS, Adkin AL. Kinematic and kinetic validity of the

802 inverted pendulum model in quiet standing. Gait \& posture. 2004;19(2):124-32.

803 59. Jayakaran P, Johnson GM, Sullivan SJ. Postural control in response to altered

804 sensory conditions in persons with dysvascular and traumatic transtibial

805 amputation. Archives of Physical Medicine and Rehabilitation. 2015;96(2):331-9.

806 60. Tschiedel M, Russold MF, Kaniusas E. Relying on more sense for enhancing lower

807 limb prostheses control: a review. Journal of NeuroEngineering and Rehabilitation.

$8082020 ; 17(1): 1-13$. 
809 61. Zelechowski M, Valle G, Raspopovic S. A computational model to design neural

810 interfaces for lower-limb sensory neuroprostheses. Journal of neuroengineering and

811 rehabilitation. $2020 ; 17(1): 1-13$.

812 


\section{Figures}
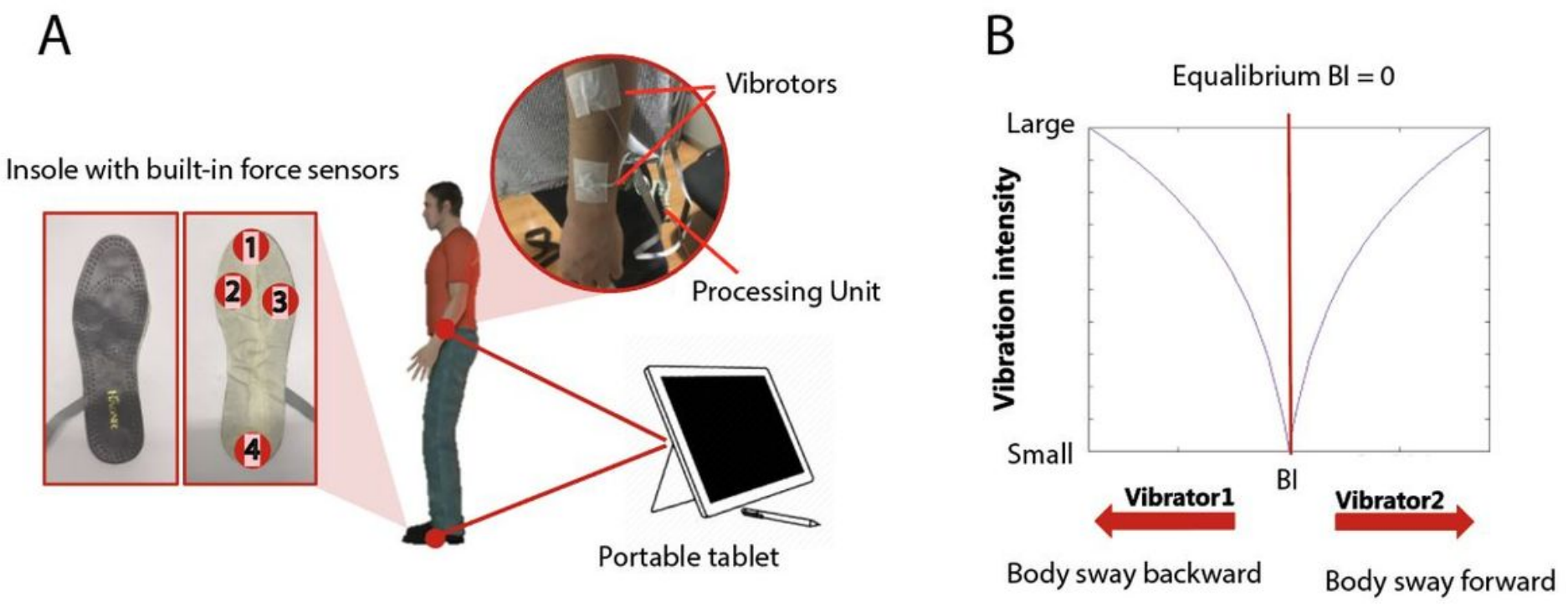

Figure 1

Illustration of the sensory substitution system. (A) The insole is instrumented with four thin electropiezo force sensors whose measurements are routed to a tablet for real-time data processing. The measured force determines the vibration intensity of the two tactile vibrators attached along the forearm's longitudinal axis on the same side of the insole. When participants walk or stand still, the vibration provides real-time feedback of the balance performance from the measured foot. (B) The vibration intensity follows a logarithmic relationship with the balance index $(\mathrm{BI})$, which is determined by the changing force loading caused by body sway. Forward or backward body lean would lead to one vibrator working, respectively. 


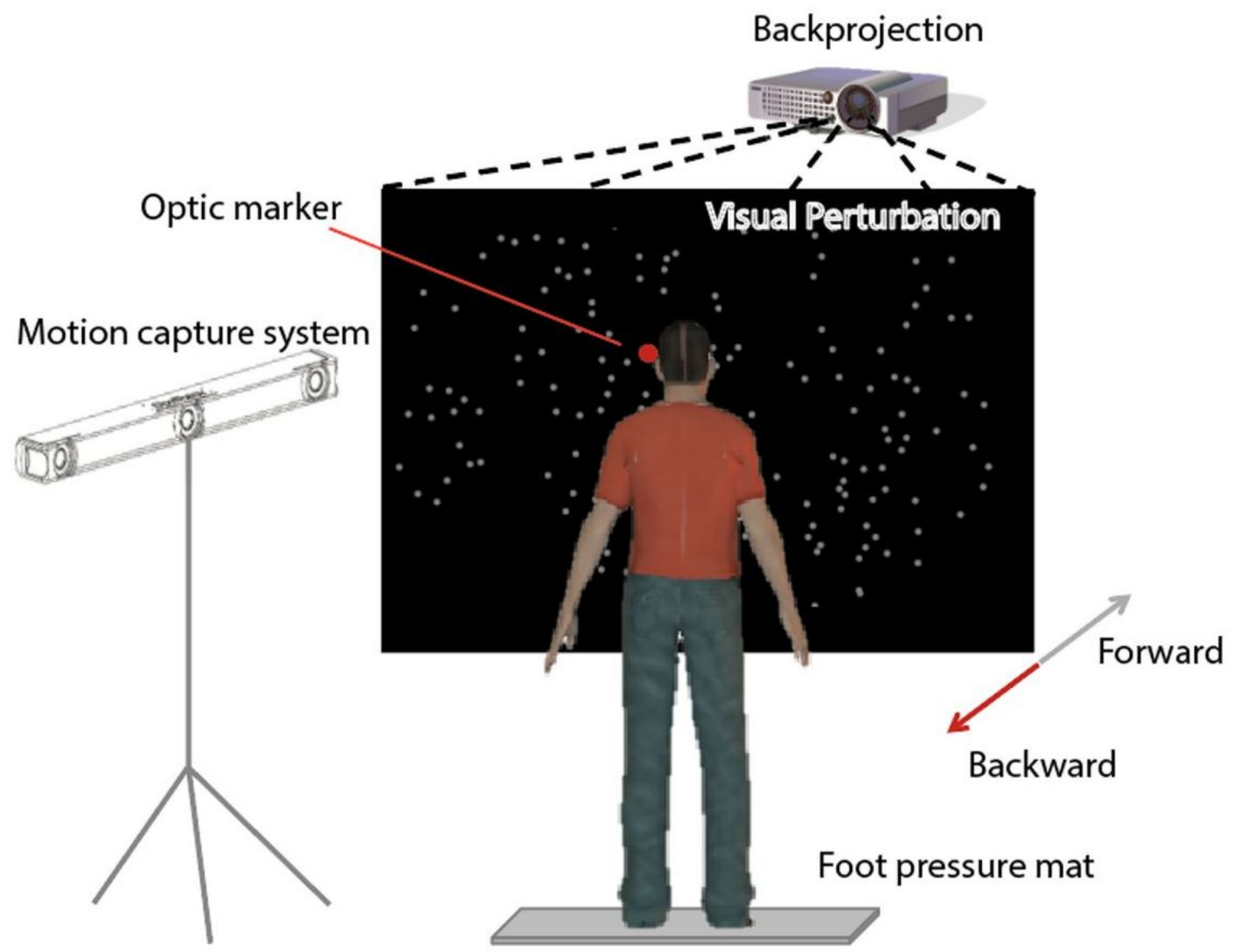

Figure 2

Illustration of the experimental setup for the postural stability test. The participant stands on a plantar pressure mat, facing a large projection screen. The field of view is limited to the screen by asking the participant to wear a pair of goggles. The head motion is simultaneously tracked by a motion tracking system with a marker placed at the eye level. The screen displays a cloud of random dots with simulated motion in the depth direction to perturb the standing posture in the anteroposterior direction. 
A

CoP in AP direction

$\mathrm{BI}$

Vibration intensity
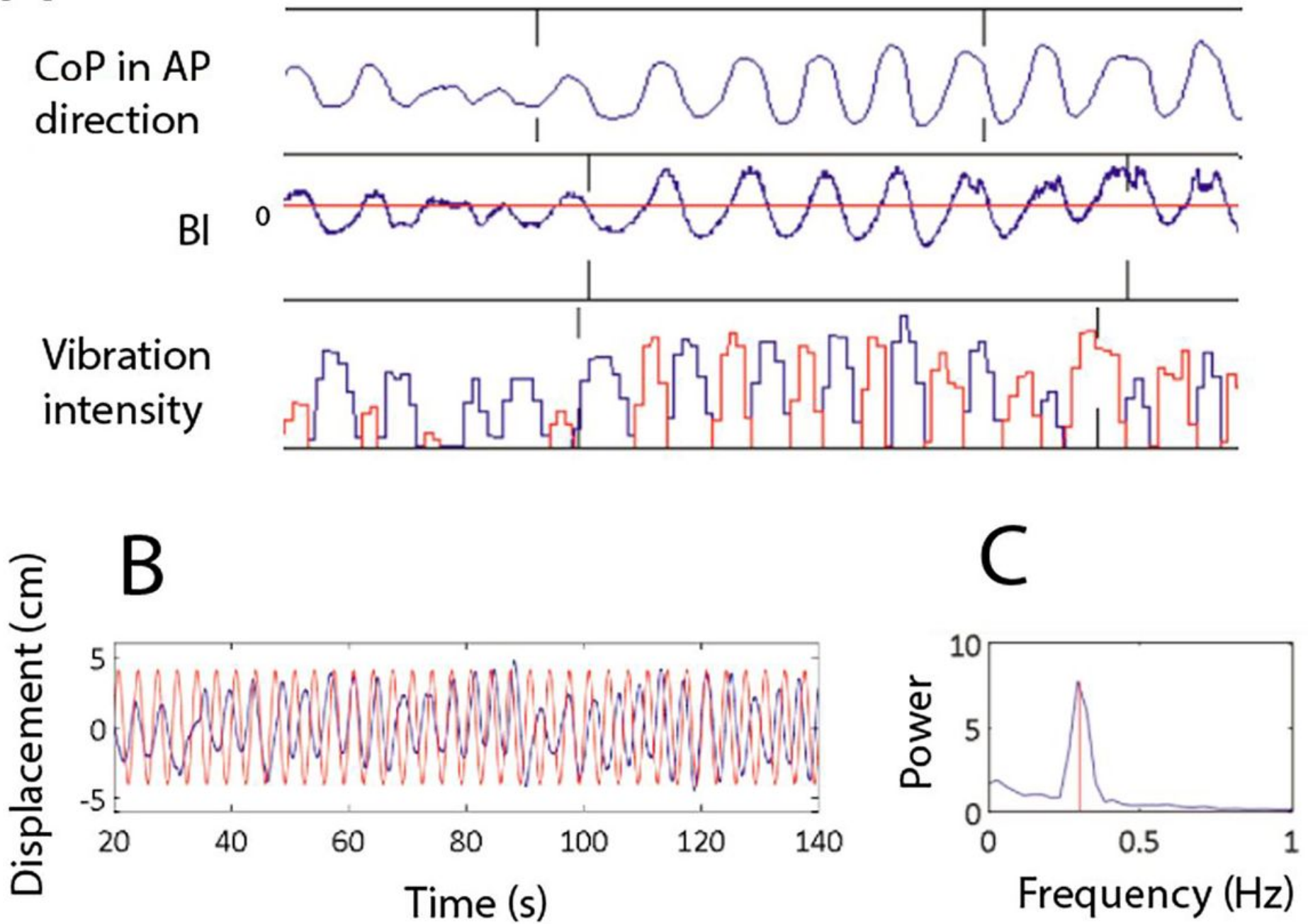

Figure 3

Exemplary data from the moving room paradigm. A) An exemplary trial segment to show how the sensory substitution system works. The participant is perturbed by the oscillatory visual stimuli, resulting in large CoP displacement in the anteroposterior (AP) direction. Our system computed the BI index in realtime and changed the vibration intensity of the two vibrators (shown in blue and red, respectively) placed on the forearm of the participant. B) An exemplary trial with head displacement (blue) and visual stimulus displacement (red) in the anteroposterior direction. The formal data collection begins at the 20th second. C) The power spectrum of head movement data of the same trial. The frequency of the visual stimulus here is $0.3 \mathrm{~Hz}$. The integral of the power over the frequency range between 0 and $2 \mathrm{~Hz}$ was used for evaluating postural stability. 
Control

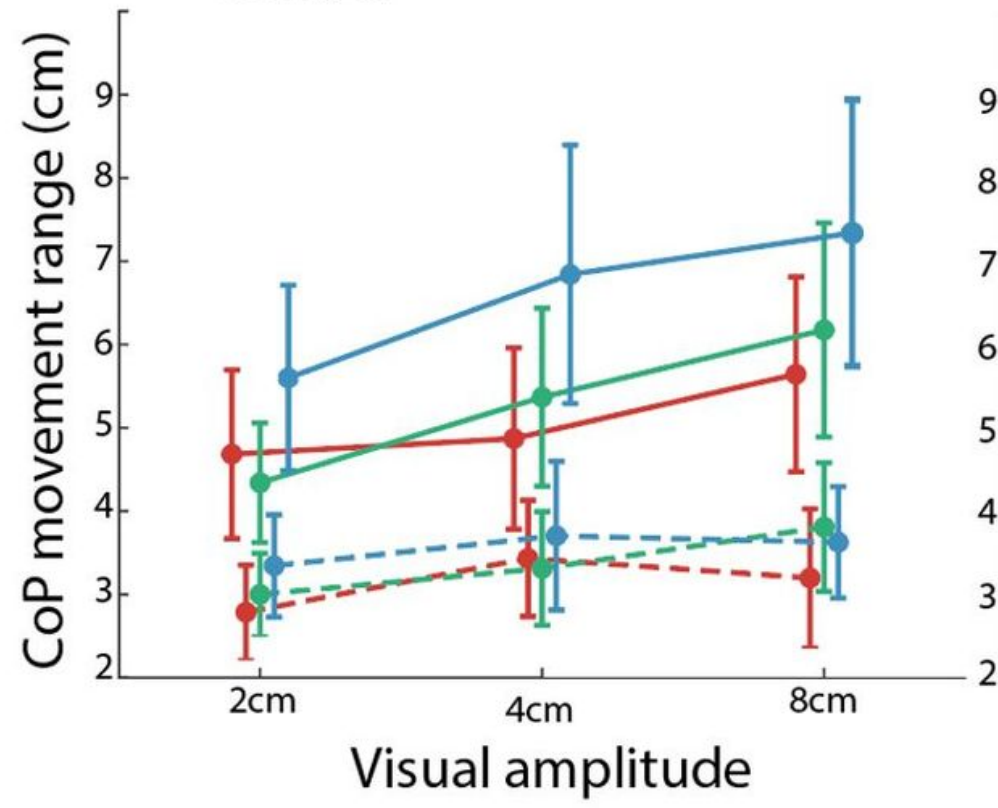

Amputee

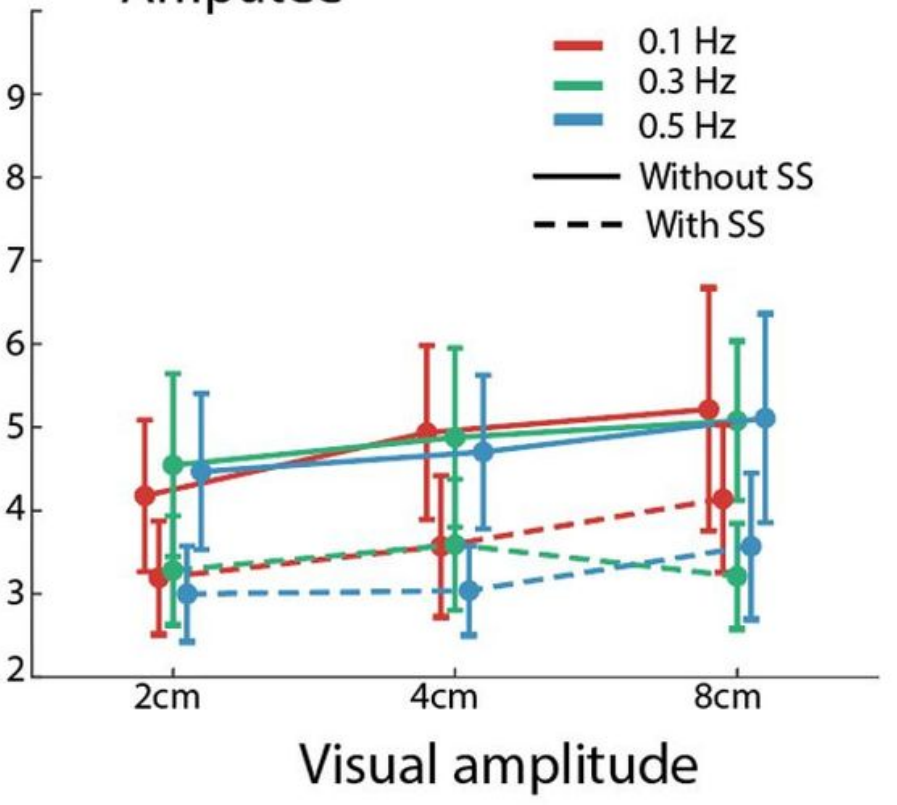

\section{Figure 4}

The range of CoP displacement in the anteroposterior direction plotted as a function of stimulus amplitude and frequency. The conditions with and without sensory substitution (SS) are shown in separate lines. The able-bodied control group and the amputee group are shown in the left and right panels, respectively.
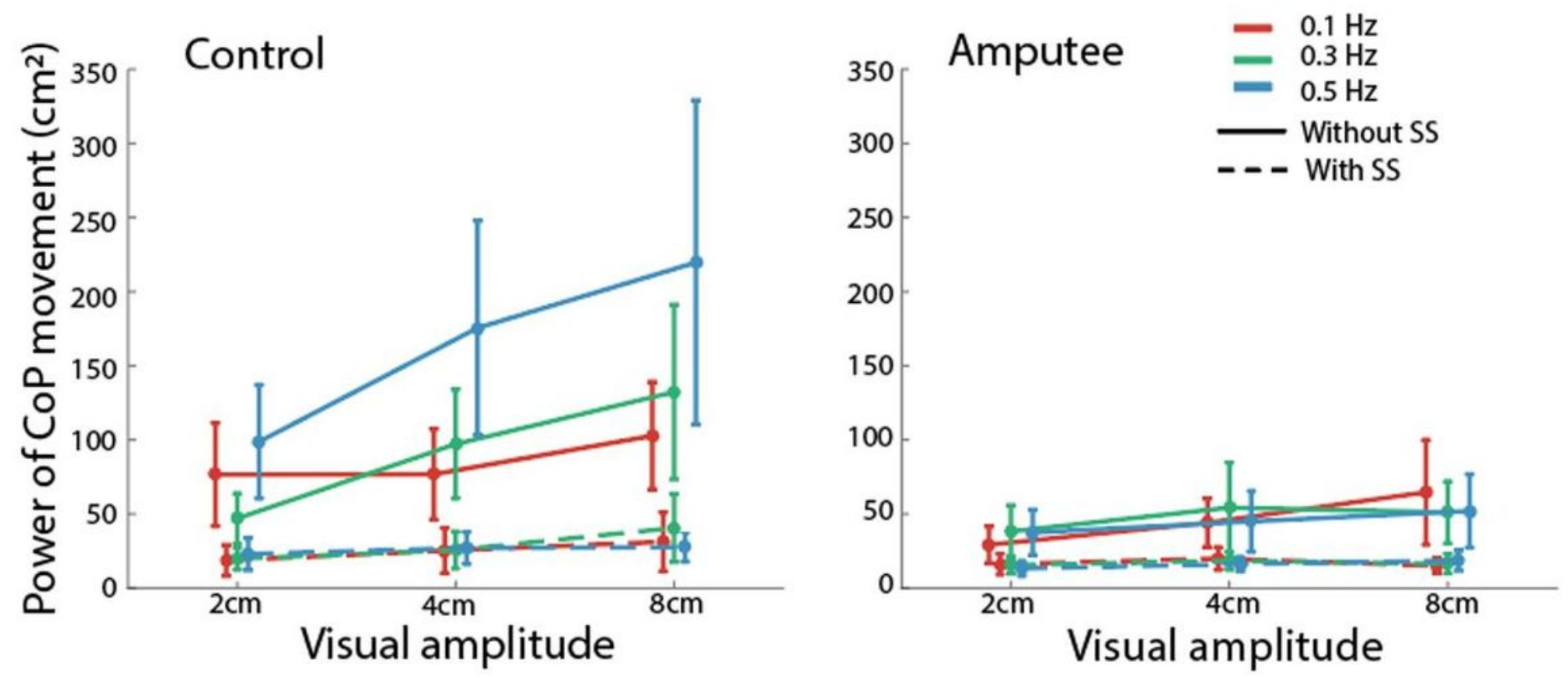

Figure 5 
The power of CoP displacement plotted as a function of stimulus amplitude and frequency. The conditions with and without sensory substitution (SS) are shown in separate lines. The able-bodied control group and the amputee group are shown in the left and right panels, respectively.
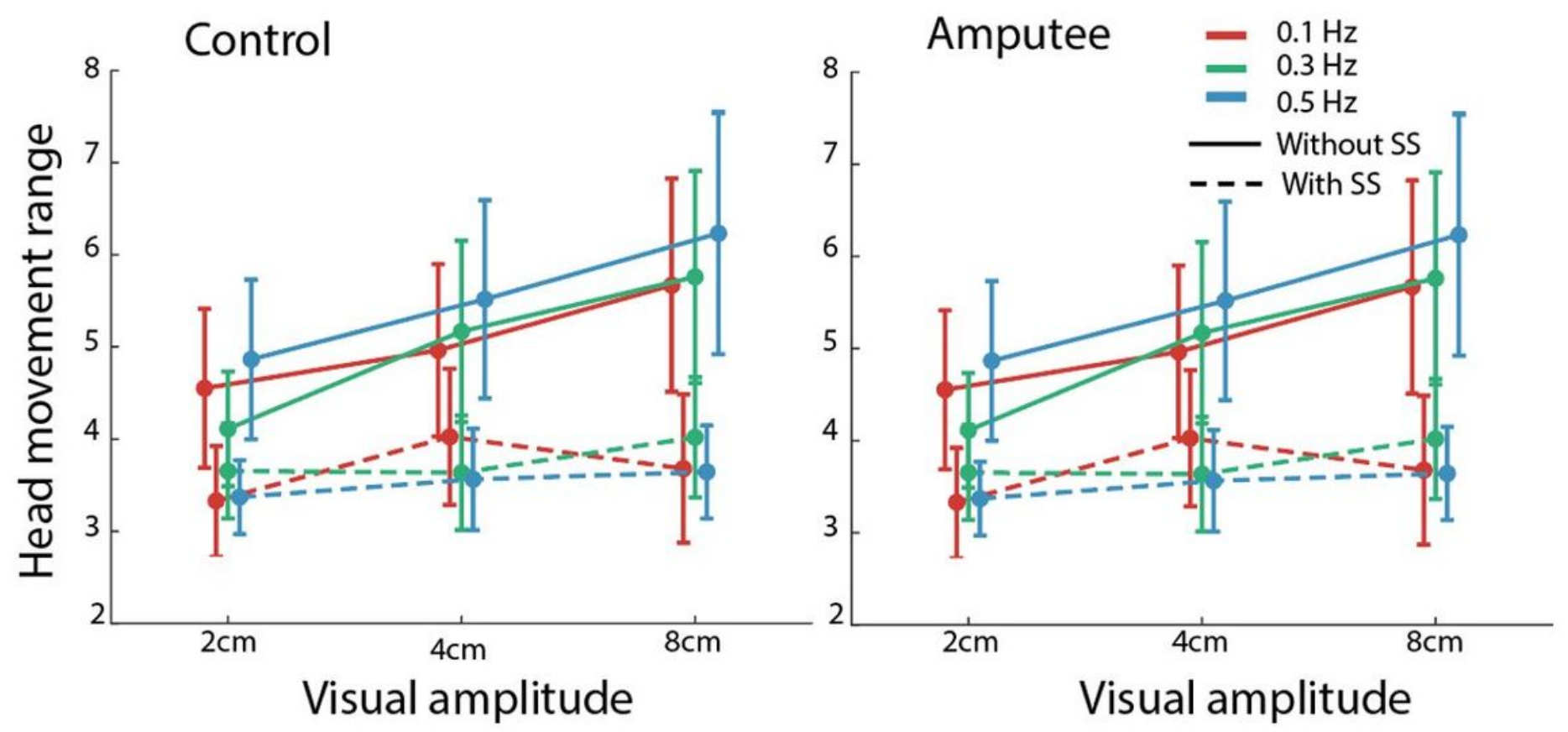

Figure 6

The head movement range in the anteroposterior direction plotted as a function of stimulus amplitude and frequency. The conditions with and without sensory substitution (SS) are shown in separate lines. The able-bodied control group and the amputee group are shown in the left and right panels, respectively. Note the head movement range is unit-less as it is normalized by dividing the participant's body height.
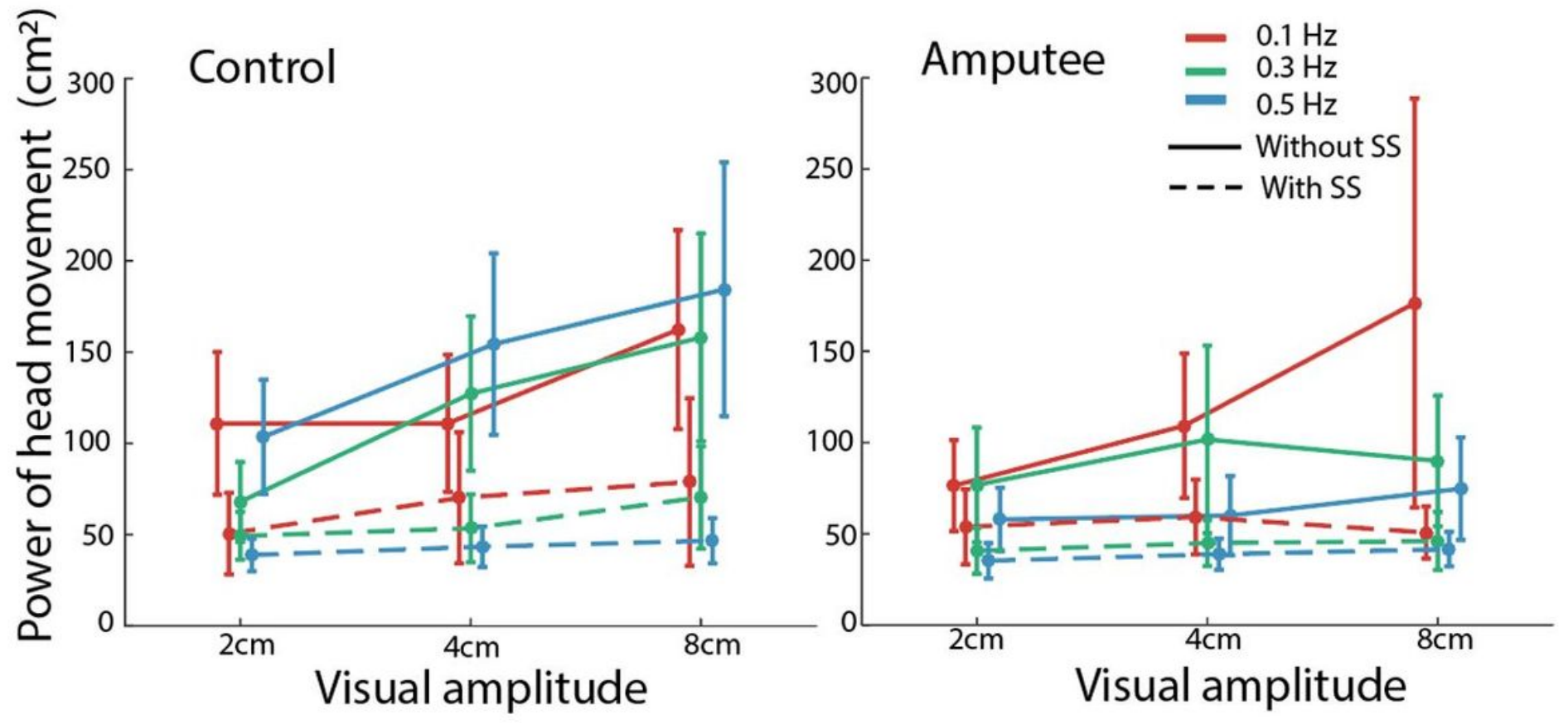


\section{Figure 7}

The power of head movement plotted as a function of stimulus amplitude and frequency. The conditions with and without sensory substitution (SS) are shown in separate lines. The able-bodied control group and the amputee group are shown in the left and right panels, respectively. 